Aufsatz

Hannes Adomeit*

\title{
Russland und der Westen: Von „strategischer Partnerschaft" zur strategischen Gegnerschaft
}

https://doi.org/10.1515/sirius-2021-2002

Kurzfassung: Gegen Ende der Jelzin-Ära erhoben Deutschland und die EU Russland $\mathrm{zu}$ ihrem „strategischen Partner“. Diese Charakterisierung der Beziehungen wurde von den beiden Akteuren nicht als Teil einer entfernten Vision, sondern als vollendete Tatsache betrachtet. Entsprechend sollten die mit Putins Russland vereinbarten „Modernisierungspartnerschaften“ lediglich als Ausfüllung der „strategischen“ Partnerschaften dienen. Derartige Vorstellungen waren schon zum Zeitpunkt ihrer Verkündung realitätsfremd. Die Beziehungen zwischen Russland und dem Westen führten von der Illusion einer strategischen Partnerschaft zur Wirklichkeit einer strategischen Gegnerschaft. Diese Entwicklung ist nicht als zusammenhanglose Anhäufung von Konflikten, geschweige denn Missverständnissen und Fehlwahrnehmungen zu verstehen, sondern als eine prinzipielle, nachhaltige und innenpolitisch in der Machtstruktur des Systems Putin verankerte und von der Moskauer Machtelite bewusst herbeigeführte Orientierung. Dies stellt eine strategische Realität dar, die eine entsprechende Antwort der westlichen Staatengemeinschaft verlangt.

Schlüsselbegriffe: Russland, Deutschland, strategische Gegnerschaft, strategische Partnerschaft, Rivalität in den internationalen Beziehungen, Putin-Regime

Abstract: Towards the end of the Yeltsin era, Germany
and the EU elevated Russia to their "strategic partner".
That characterization was meant by the two actors not
to reflect a distant vision, but it was understood to be an
accomplished fact. The "modernization partnerships"
that they concluded with Putin's Russia accordingly were
held to provide substance to the allegedly existing "stra-
tegic" partnerships. Such ideas may have conformed to
the precepts of Western transition theory, but they were
out of touch with reality even at the time when they were

*Kontakt: Dr. Hannes Adomeit, Senior Fellow, Institut für Sicherheitspolitik an der Universität Kiel, Büro Berlin;

E-Mail: hannes-adomeit@t-online.de announced. The relationship between Russia and the West went from the illusion of strategic partnership to the reality of strategic confrontation. This development is not to be interpreted as a disjointed accumulation of conflicts, let alone misunderstandings and misperceptions, but as a fundamental orientation, firmly anchored in the power structure of the Putin system and consciously brought about by the Moscow power elite. This state of affairs represents a strategic reality that requires a strategic response from the Western community of states.

Keywords: Russia, Germany, strategic confrontation, strategic partnership, rivalry in international politics, Putinregime

\section{Einleitung}

Gegen Ende der Jelzin-Ära erhob das Auswärtige Amt Russland zu Deutschlands „strategischem Partner“. ${ }^{1}$ Mit Deutschland im Vorsitz im Europäischen Rat wurde Russland auch zum „strategischen Partner“ der EU stilisiert - nicht als Teil einer entfernten Vision, sondern als vollendete Tatsache. ${ }^{2}$ Offensichtlich in Anbetracht der sich immer schwieriger gestaltenden Beziehungen und nur drei Monate vor Russlands Krieg in Georgien meinte Außenminister Frank-Walter Steinmeier in einer Rede in Jekaterinburg: „Die strategische Partnerschaft braucht eine umfassende und verlässliche Grundlage.“ Dazu sollte die Gründung einer deutsch-russischen „Modernisie-

1 Auf der Website des Auswärtigen Amts gelangte man von der „Außenpolitik“ zu den „Regionalen Schwerpunkten“ und dann zur „Strategischen Partnerschaft mit Russland“; http://www.auswaertigesamt.de/DE/Aussenpolitik/RegionaleSchwerpunkte/Russland/Russ land_node.html.

2 In der Gemeinsamen Strategie der Europäischen Union gegenüber Russland von 1999 heißt es: „Der Europäische Rat nimmt [...] diese Gemeinsame Strategie zur Festigung der strategischen Partnerschaft zwischen der Europäischen Union und Russland am Beginn eines neuen Jahrhunderts an. [...] Die Europäische Union schlägt vor, dass die strategische Partnerschaft im Rahmen eines permanenten politischen und sicherheitspolitischen Dialogs weiterentwickelt wird.“ 
rungspartnerschaft" dienen. ${ }^{3}$ In der Tat bemühte sich die Bundesregierung, dieses Projekt auszufüllen. Desgleichen auch die EU. So verpflichteten sich die beiden Akteure als „langjährige strategische Partner“ auf der Gipfelkonferenz in Rostow am Don im Juni 2010, gemeinsamen Herausforderungen zu begegnen. Dem deutschen Vorbild entsprechend, gründeten sie eine „EU-Russland Modernisierungspartnerschaft" “. ${ }^{4}$ Erst nach Russlands Annexion der Krim und seiner Aggression in der Ostukraine verschwand der Begriff der strategischen Partnerschaft aus dem offiziellen Sprachgebrauch sowohl Deutschlands als auch der EU.

Das Europäische Parlament nahm dabei eine Vorreiterrolle ein. So stellte es in einer Resolution vom 10. Juni 2015 fest, Russland könne „aufgrund seiner Aktionen auf der Krim und in der Ostukraine nicht mehr als ,strategischer Partner' behandelt oder betrachtet werden [, denn] strategische Partnerschaften müssen auf gegenseitigem Vertrauen und Achtung des Völkerrechts, auf [Anerkennung demokratischer Prinzipien], staatlicher Souveränität, der Freiheit der Wahl der inneren Verfassungsordnung sowie der außenpolitischen Orientierungen, der territorialen Integrität des Staates, Rechtstaatlichkeit, Menschenrechten und Grundsätzen der internationalen Diplomatie und des internationalen Handels beruhen “. ${ }^{5}$ Nach Überzeugung der großen Mehrheit der Mitglieder des Parlaments hatte das Russland Putins all diese Prinzipien mit Füßen getreten.

Was aber soll konzeptionell und begrifflich die offensichtlich unhaltbare Sprachregelung von Russland als „strategischer Partner“ ersetzen? Wenn Worte nicht bedeutungslose Leerformeln sein sollen, ist dies eine Frage, die nicht nur für Wissenschaftler und Journalisten, sondern auch und gerade für Politiker wichtig ist. Im Juni 2019 hat der Außendienst der EU in seiner Globalen Strategie

\footnotetext{
3 Rede von Außenminister Frank-Walter Steinmeier am Institut für internationale Beziehungen der Ural-Universität in Jekaterinburg: „Für eine deutsch-russische Modernisierungspartnerschaft“, Auswaertiges-amt.de, 13.5.2008, https://www.auswaertiges-amt.de/de/ newsroom/080513-bm-russland/219750.

4 "The European Union and Russia, as long-standing strategic partners in a changing multipolar world, are committed to working together to address common challenges [...]." Council of the European Union: Joint Statement on the Partnership for Modernisation, EU-Russia Summit, 31 May-1 June 2010, Consilium.europa.eu, 1.6.2010, https://www.consilium.europa.eu/uedocs/cms_data/docs/ pressdata/en/er/114747.pdf.

5 Resolution of 10 June 2015 on the state of EU-Russia relations, European Parliament, Europarl.europa.eu, 10.06.2015, https:// www.europarl.europa.eu/doceo/document/TA-8-2015-0225_EN. html und https://www.europarl.europa.eu/news/en/press-room/ 20150604IPR62878/russia-is-no-longer-a-strategic-partner-of-the-eusay-meps. Die Resolution wurde mit 402 gegen 163 Stimmen bei 89 Enthaltungen angenommen.
}

darauf eine Antwort gegeben. Das Verhältnis zu Russland wird dort nicht mehr als strategische Partnerschaft, sondern als „strategische Herausforderung“ bezeichnet. ${ }^{6}$ Dieser Begriff wird allerdings der tiefen Kluft in den Beziehungen nicht gerecht. Er lässt die tatsächlichen Verhältnisse in einem weicheren Licht erscheinen. Treffender ist es, von einer strategischen Gegnerschaft zu sprechen. Warum, wird im Folgenden weiter ausgeführt.

\section{Wahrnehmungen und Wirklich- keit strategischer Gegnerschaft}

Strategische Gegnerschaft - so die dieser Analyse zu Grunde gelegte Definition - liegt dann vor, wenn ein Staat das Ziel anstrebt, die globalen Machtverhältnisse grundlegend $\mathrm{zu}$ verändern, die internationale, regelbasierte Ordnung zu unterhöhlen und die diese tragenden politischen Kräfte zu schwächen. Sie schließt die Gefahr einer größeren militärischen Auseinandersetzung mit ein und beinhaltet daher existenzielle Risiken für die Länder des Westens. Eine strategische Gegnerschaft kann sich innerhalb eines Kontinuum abspielen. Dieses kann von einem „heißen“ bis zu einem „kalten“ Krieg spannen und kann auch in einer politischen Übereinkunft enden, wie sie in den Jahren 1989/90 erzielt wurde. ${ }^{7}$

Das Bewusstsein, dass das Verhältnis zwischen Deutschland und Russland der hier gebrauchten Definition von „Strategischer Gegnerschaft“ entspricht, ist in Teilen der deutschen Öffentlichkeit gewachsen. Das gilt auch für die Bundesregierung. So hat Heiko Maas in seinem ersten Interview nach Übernahme des Außenministeriums festgestellt, dass Russland ein „zunehmend feindseliges“ Verhalten aufweise. Es habe „erstmals seit Ende des Zweiten Weltkriegs mitten in Europa geächtete chemische Waffen eingesetzt“. Cyberangriffe schienen zu einem integralen „Bestandteil russischer Außenpolitik“ zu werden“. In einem so schwerwiegenden Konflikt wie in Syrien habe Russland den „Uno-Sicherheitsrat blockiert“ und in der Ukraine eine

6 The European Union's Global Strategy: Three Years on, Looking Forward, Eeas.europa.eu [June 2019], https://eeas.europa.eu/sites/ eeas/files/eu_global_strategy_2019.pdf. Aus dem Debakel mit der Begrifflichkeit der „strategischen Partnerschaft“ mit Russland hatte der Außendienst der EU offensichtlich nichts gelernt. In der Globalen Strategie wird das Verhältnis zu China als „umfassende strategische Partnerschaft" bezeichnet.

7 Diese Definition folgt einem noch unveröffentlichten Papier der Stiftung Wissenschaft und Demokratie zum Thema „Globaler Wandel und deutsche Außenpolitik“, welches im Sommer 2021 erscheinen wird. 
„Aggression“" verübt. ${ }^{8}$ Russlands Krieg in Syrien nahm er zudem im Sicherheitsrat der Vereinten Nationen zum Anlass, die russische Luftwaffe für „Kriegsverbrechen“ verantwortlich zu machen. Die Regierung von Damaskus und ihr Verbündeter Russland seien ,verpflichtet, die Zivilbevölkerung zu schützen [aber sie bombardieren stattdessen] die zivile Infrastruktur wie Krankenhäuser und Schulen“.9

„Gegnerschaft“ ist terminologisch wie auch in der Wirklichkeit mit verdeckter oder offener Kriegführung verbunden. So stellte Bundeskanzlerin Angela Merkel am 13. Mai 2020 im Bundestag fest: „Es gibt eine Strategie Russlands der hybriden Kriegsführung. Das dürfen wir nicht verdrängen. Das ist Kriegsführung in Form von Cyber-Desorientierung und Desinformation. Das ist eine Strategie, kein Zufallsprodukt.“ In diesem Zusammenhang wies sie auf den Hackerangriff auf ihr eigenes Büro und das anderer Abgeordneter hin. Die Ermittlungen des Generalbundesanwalts hätten „harte Evidenzen“ für eine russische Beteiligung ergeben. Dass die Spuren des Angriffs im Jahr 2015 zum russischen Geheimdienst führten, so meinte sie, sei „ungeheuerlich“. ${ }^{10}$

Den Begriff der hybriden Kriegsführung hat auch der Bundestagsabgeordnete Alexander Graf Lambsdorff (FDP) gebraucht. „Die Machthaber im Kreml haben sich vom Frieden verabschiedet und führen einen kalten, einen hybriden Krieg gegen den Westen, gegen Europa, gegen unsere Werteordnung. Die Machthaber im Kreml wollen die Landkarte Europas verändern, zur Not mit Gewalt. Der Kreml führt heiße Kriege in der Ostukraine und in Syrien. Ob in Moldawien, Georgien oder auf der Krim, Moskau stationiert immer wieder Soldaten auf dem Gebiet anderer Länder gegen den Willen der dortigen Regierungen." ${ }^{11}$

Dass es sich bei den unterschiedlichen „feindseligen“ und „ungeheuerlichen“ Handlungen nicht um bedauerliche Einzelfälle handle, sondern um ein Verhaltensmuster und integrale Bestandteile einer Strategie, hat auch die heutige EU-Kommissionspräsidentin Ursula von der Leyen in ihrer damaligen Eigenschaft als Bundesverteidigungsministerin festgestellt. „Denen, die engere Beziehungen zu Russland befürworten, sage ich, dass die Vergiftung von Alexej Nawalny mit einem weit entwickelten chemischen

8 „Syrien ist nicht Auschwitz“ - Spiegel-Gespräch, Der Spiegel, 16/ 2018 [Hervorhebung nicht im Original].

9 Germany says Syria and Russia responsible for Idlib „war crimes“, Deutsche Welle, 27.02.2020.

10 Daniel Schreckenberg/Ulricht Diekmann: „Ungeheuerlich“ - Merkel macht Russland schwere Vorwürfe. Schlagabtausch im Bundestag, T-online.de, 13.5.2020, [Hervorhebungen nicht im Original].

11 Deutscher Bundestag, 19. Wahlperiode, 208. Sitzung, 10. Februar 2021, S. 26243-26244, Bundestag.de, 10.02.2021, https://dserver. bundestag.de/btp/19/19208.pdf [Hervorhebung nicht im Original].
Wirkstoff kein Einzelfall ist. Wir haben das Muster [derartiger Aktionen] in Georgien und der Ukraine, in Syrien und in Salisbury gesehen - und bei der Einmischung in Wahlen weltweit. Dieses Muster ändert sich nicht, und kein Stopp einer Pipeline wird daran etwas ändern."12

Auch Michael Roth, Staatsminister im Auswärtigen Amt, hat die Hackerangriffe, den mutmaßlichen Auftragsmord an einem Georgier im Berliner Tiergarten und gezielte Desinformationskampagnen - „die Liste ist lang“ - als Teil eines gegen Deutschland und den Westen gerichteten feindseligen Verhaltensmusters gewertet: „Moskau definiert sich immer mehr in Abgrenzung und teilweise in Gegnerschaft zur liberalen Demokratie und zum ,Westen'. [...] Auch gegenüber der EU tritt Russland zunehmend aggressiv auf. [...] Die russische Führung lässt kaum eine Gelegenheit für Versuche aus, einen Keil zwischen uns zu treiben. [Sie] betreibt gezielt die Bilateralisierung der Beziehungen. [...] Das aggressive Auftreten Moskaus nach außen geht einher mit wachsender Repression im Inneren."13 Das emotionsgeladene Wort, wenn auch wie bei Roth mit einschränkendem Adjektiv versehen, ist auch vom CDU-Vorsitzenden Armin Laschet benutzt worden. Wir bräuchten Russland zwar bei „wichtigen Themen“; ,in vielen Belangen“ sei Russland aber „ein Gegner“. ${ }^{14}$

Am nächsten kommt das Verteidigungsministerium dem Begriff (und den Inhalten) der Gegnerschaft: „Russland definiert sich als Gegenmacht zum Westen. Immer deutlicher hat Moskau seine militärischen und politischen Drohungen in jüngster Zeit verschärft und internationale Verträge wissentlich verletzt. Russland wendete in den vergangenen Jahren in seiner Nachbarschaft militärische Gewalt an und rüstet massiv konventionell und nuklear auf. Aus dieser Lage ergeben sich sehr konkrete Bedrohungen für Deutschland." ${ }^{15}$

Gehört zu den in der Ära Putin veränderten Wahrnehmungen auch die Erkenntnis, dass Russlands Gegnerschaft zum Westen - wie im Kalten Krieg - eine ideologische Dimension besitzt und Systemkonkurrenz beinhaltet? Dass dies der Fall ist, darauf deuten zumindest Charakterisierungen des Systems Putin als autoritär oder autokratisch hin, dass es sich immer weiter von demokratischen und

12 State of the Union Address by President von der Leyen at the European Parliament, Ec.europa.eu/commission, 16.09.2020, https:// ec.europa.eu/commission/presscorner/detail/en/SPEECH_20_1655 [Hervorhebung nicht im Original].

13 Michael Roth: Unser Verhältnis zu Russland: Die Chancen nach dem Tiefpunkt, Spiegel Online 31.1.202.

14 Laschet fordert Nawalnys „sofortige Freilassung“, Spiegel Online, 22.01.2021 [Hervorhebung nicht im Original].

15 Bundesministerium der Verteidigung 2021. 
rechtstaatlichen Prinzipien entfernt habe und dass es die regelbasierte internationale Ordnung untergraben wolle.

Im Regierungslager mangelt es jedoch an einer klaren, auch öffentlichkeitswirksamen Darstellung der Verbindung von inneren und externen systempolitischen Dimensionen in den Beziehungen des Kremls zum Westen. Auf diesen Zusammenhang hat dagegen Michael Brand (CDU) hingewiesen. „In Russland hat der ehemalige KGBAgent an fast allen wichtigen Schaltstellen des Staates und der Wirtschaft alte, korrupte Kameraden installiert“, stellte er einleitend in einer Bundestagsdebatte fest. „Was noch gefährlicher ist als diese inneren Entwicklungen, ist aber Putins unerklärter Krieg gegen Demokratie und Menschenrechte in seinem eigenen Land und Putins digitaler Krieg gegen die westlichen Demokratien. Es geht um einen, wie es im militärischen Jargon heißt, ,all-out war', um einen ,totalen Krieg' gegen die Demokratie [...]. " "16

Westliche Regierungen haben lange gebraucht, bis sie ein derartiges Bewusstsein entwickelt haben. ${ }^{17}$ Immer wieder hat die Bundesregierung fast bis zur Selbstverleugnung auf Provokationen und aggressives Verhalten Moskaus mit der Suche nach verbliebenen gemeinsamen Interessen und mit Dialogangeboten reagiert. ${ }^{18}$ Ähnliches lässt sich auch, wenn auch mit erheblichen Einschränkungen, für die Vereinigten Staaten vor der Amtszeit Donald Trumps sagen. Immer wieder, wenn eine neue Regierung in Washington ins Amt kam, bemühte sie sich um einen Neustart, einen reset der Beziehungen, und immer wieder hatte sich am Ende der jeweiligen Amtszeit das russischamerikanische Verhältnis verschlechtert. ${ }^{19}$ Im Präsidentschaftswahlkampf 2012 hatte Republikaner Mitt Romney zwar den Standpunkt vertreten, Russland sei ohne Frage „geopolitischer Feind Nummer Eins“ der Vereinigten Staaten, er wurde aber dafür scharf kritisiert. ${ }^{20}$ So merkte

16 Deutscher Bundestag, 19. Wahlperiode, 208. Sitzung, 10. Februar 2021, op. cit: 26239.

17 Laut James Forsyth, Leiter des Politik-Referats des „Spectators“, war Boris Johnson bei seiner Ernennung zum Außenminister überzeugt, dass er einen reset in den Beziehungen zu Moskau bewerkstelligen könnte. Aus den Salisbury-Vergiftungen hätte er aber den Schluss gezogen, dass Russland kein Interesse daran hätte, sich zu ändern: „Johnson - nicht gerade der intellektuellen Selbstreflexion oder dem Eingestehen von Fehlern geneigt - sagte mir [...-], dass einer seiner größten Fehler als Außenminister darin bestand, zu glauben, er könne Russland aus der Kälte hereinbringen" [Hervorhebung nicht im Original]. James Forsyth: „Biden victory is a mixed blessing for Britain“, The Times online, 5.11.2020.

18 Reinhard Veser: Dialog mit Russland: Sich nicht von Lawrow beeindrucken lassen, Faz.net, 15.10.2020.

19 Die Rekonstruktion dieser Zyklen bei Adomeit 2017c.

20 Romney benutzte das Wort foe. Dies ist gleichlautend mit enemy (Feind), allerdings weniger scharf im Ton.
Barack Obama, Präsidentschaftskandidat und Konkurrent Romneys, sarkastisch an: „Jetzt melden sich die 1980-er Jahre und wollen ihre Außenpolitik zurück haben.“21

Allerdings entschuldigte sich Madeleine Albright, Außenministerin in der Regierung Bill Clintons, die Präsident Obama in den Präsidentschaftswahlen 2012 unterstützt hatte, später bei Romney: „Wir haben unterschätzt, was in Russland vor sich ging. “22 Noch deutlicher wurde der republikanische Senator John McCain, der 2017 sagte: "Jeder unserer letzten drei Präsidenten hatte große Hoffnungen, mit der russischen Regierung eine Partnerschaft aufzubauen. Jeder Versuch scheiterte, [allerdings] nicht aus Mangel an gutem Willen und Bemühungen auf USamerikanischer Seite, sondern weil Putin unser Feind sein will. Er braucht uns als sein Feind. Er wird niemals unser Partner sein." ${ }^{23}$ Und im Wahlkampf 2020 schloss Joe Biden, der ehemalige Vizepräsident unter Obama, unausgesprochen den Bogen zu McCain und Romney: „Ich denke, dass Russland derzeit die größte Bedrohung für Amerika hinsichtlich der Auflösung unserer - unserer Sicherheit und unserer Allianzen ist.“24

Diese Charakterisierung entspricht schon nicht mehr den tatsächlichen Gegebenheiten. Schon die Nationale Sicherheitsstrategie 2018 stellte fest: "Zwischenstaatliche strategische Konkurrenz, nicht [internationaler] Terrorismus, ist derzeit die hauptsächliche Herausforderung für die nationale Sicherheit der Vereinigten Staaten.“ China und Russland werden - in dieser Reihenfolge - als ,revisionistische Mächte“ und „langfristige strategische Konkurrenten“ der USA bezeichnet. ${ }^{25}$

21 Cheyenne Haslett: Mitt Romney finally gets credit years later for his warnings on Russia, Abcnews.go.com, 26.2.2019.

22 Ebd.

23 Die Aussage findet sich auf der noch erhaltenen Facebook-Präsenz des inzwischen verstorbenen Politikers, https://www.facebook. com/johnmccain/posts/10155019644158707.

24 Mark Moore: Trump, Biden clash when asked about biggest foreign threat to US, New York Post online 26.10.2020 [Hervorhebung nicht im Original]. In der Senatsanhörung zur Ernennung als Außenminister wandte sich Tony Blinken zum anwesenden Senator Mitt Romney und machte ihm das Kompliment, er sei „,vorausschauend“ gewesen, Russland als geopolitischen Feind Nummer Eins der USA zu bezeichnen. Dominic Lawson: Germany, not Brexity Britain, will vex Biden. Berlin's desire to appease Russia and China won't endear it to the president, The Times online, 24.1.2021.

25 U.S. National Defense Strategy 2018, für das Bundesverteidigungsministerium ist China zwar von einer aufstrebenden Volkswirtschaft „zu einem machtvollen und immer häufiger sichtbar ausgreifenden Akteur geworden“. Unklar ist damit aber, ob China die primäre Herausforderung für Deutschland oder den Westen darstellt, Bundesministerium der Verteidigung 2021. 
Wahrnehmungen sind eine Sache. Wie aber sieht es mit der Wirklichkeit aus? Welche Entwicklungen rechtfertigen die Klassifizierung des Verhältnisses Russlands zum Westen als strategische Gegnerschaft? Antworten darauf finden sich im „Tandem“-Intervall an der Führungsspitze des Kremls, dem Ämtertausch zwischen Putin und Medwedew im Zeitraum von 2008-2012. ${ }^{26}$

\section{Sozioökonomische Modernisie- rung mithilfe des Westens}

Die Jahre zwischen 2008 und 2012 standen anfangs im Zeichen sozioökonomischer Modernisierung Russlands mithilfe des Westens. Die Phase wurde aber am Ende des Ämtertauschs und mit Beginn der dritten Amtszeit Putins ziemlich rasch durch national-patriotische Mobilisierung und den Wunsch nach Konfrontation mit dem Westen abgelöst - eine Entscheidung der Machthaber im Kreml zur Gegnerschaft, die bis heute im russischen Herrschaftssystem fest verankert ist.

Am Anfang stand die Einsicht der Kreml-Herrscher, dass Russland sich in einer Wirtschaftskrise befände. Im Februar 2008, noch vor dem Beginn der globalen Wirtschafts- und Finanzkrise, erklärte Putin, dass die „Existenz" des Landes gefährdet sei, falls keine tiefgreifende Änderung des wirtschaftspolitischen Kurses vorgenommen werde. Im Mai desselben Jahres lieferte Medwedew dazu die Begründung. Es gelte, strukturelle Defizite zu beseitigen. Diese sah er unter anderem in der „erniedrigenden“ Abhängigkeit“ der russischen Wirtschaft von Rohstoffen. Reformbedarf sah er aber nicht nur in der Wirtschaft, sondern auch in der Gesellschaft. So beklagte er die in Russland tief verwurzelte Korruption und „Kultur des Rechtsnihilismus“ sowie sein „halbsowjetisches“ und „archaisches“ Gesellschaftssystem. Dieses zu überwinden und einen „zivilisierten Staat“ aufzubauen, erfordere vor allem, „rechtsstaatliche“ Verhältnisse“ einzuführen.

Der Diagnose entsprechend stellte Medwedew sich an die Spitze einer Modernisierungskampagne. Russland ging, wie oben erwähnt, auf die Vorschläge Steinmeiers zur Gründung einer deutsch-russischen „Modernisierungspartnerschaft“ ein. Andere europäische Länder und die EU folgten diesem Beispiel. Schon in seinem viel beachteten Aufruf von 2009 unter dem Titel „Vorwärts, Russland!“ hatte er gefordert, dass sich die russische Außenpolitik in den Dienst der Modernisierung des Landes zu stellen

26 Die nachfolgende Rekonstruktion der Entwicklung ausführlich bei Adomeit 2017a und 2017b.

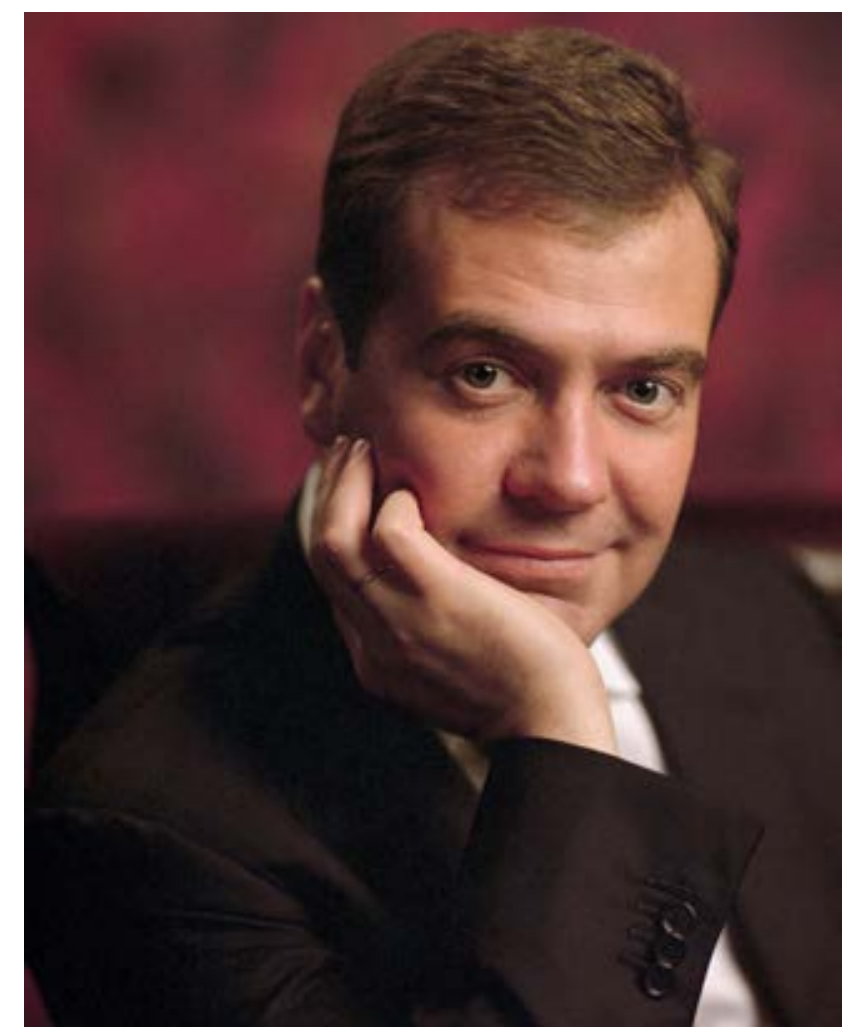

Der frühere russische Präsident Dmitri Medwedew

habe. ${ }^{27}$ Seiner Forderung entsprechend, verfasste das Außenministerium im Mai 2010 ein Grundsatzdokument unter dem wörtlich von Medwedew vorgegebenen Titel „Programm für die systematische und effektive Nutzung außenpolitischer Faktoren mit dem Ziel einer langfristigen Entwicklung Russlands“. ${ }^{28}$ Gemeint war enge Zusammenarbeit sowohl mit Europa als auch den USA.

Im Rahmen dieses Ansatzes schien sich auch die russische Politik im postsowjetischen Raum geändert zu haben. Außenminister Lawrow erklärte, Russlands Modernisierungsstrategie sei unter anderem so angelegt, die Wahrnehmungen in den Nachbarstaaten zu verändern und Russland für sie attraktiver erscheinen zu lassen. Er wiederholte zwar die russische Standardformel, dass die früheren Sowjetrepubliken der Sowjetunion ,vorrangige Partner" seien, aber in Umkehr der Formel von den privilegierten Interessen Russlands in diesem Raum sprach er davon, dass Russland das Land sei, ,auf das sich die

27 Rossija vperëd, Kremlin.ru, 19.9.2009, http://www.kremlin.ru/ news/5413.

28 Programma effektivnogo ispol'zovanija na sistemnoj osnove vnešnepolitičeskich faktorov v celjach dolgosročnogo razvitja Rossijskoj Federacii, Kremlin.ru, 11.5.2010 http://kremlin.ru/articles/ 13590.html. 
privilegierten Interessen [unserer Partner] konzentrieren.“ Westliche Beobachter folgerten, dass sich die Politik Russlands in seiner Nachbarschaft „radikal gewandelt“ hätte. Dieser Wandel hätte sich aus dem einfachen Grund vollzogen, „weil der Kreml erkannt hat, dass die frühere aggressive Politik vollkommen gescheitert ist. [...] Russlands neue Politik ist im Wesentlichen pragmatisch und auf seine nationalen Interessen fokussiert, was wiederum auf der Notwendigkeit beruht, [das Land] zu modernisieren und ausländische Investitionen anzuziehen. “29

Derartige Interpretationen stellen sich heute als verfehlt heraus. Die von Medwedew gesetzten Impulse und neuen Denkansätze verpufften nicht nur, sondern wurden aus dem öffentlichen Leben verbannt. Der öffentliche Diskurs und die darauf beruhende Politik wurden „entmedwedisiert“. Wie vorher schon der Begriff „Demokratisierung“ unter Jelzin, so strich die Kreml-Führung nunmehr den Begriff der „Modernisierung“ aus ihrem Slogan-Inventar. Wenn danach von Modernisierung die Rede war, dann praktisch nur im Zusammenhang mit den russischen Streitkräften. Wie konnte es dazu kommen?

\section{Die Legitimitätskrise}

Im Zeitraum vom Herbst 2011 bis Frühjahr 2012 wurde eine bis dahin latente Legitimitätskrise des von Putin aufgebauten autoritären Systems akut. Die Umfragewerte der Kreml-nahen Partei Einiges Russland waren in den vorangegangenen Monaten stark abgesackt - nicht zuletzt wegen der von Oppositionspolitiker und Blogger Alexej Nawalny in Umlauf gebrachten Brandmarkung der Partei als „Partei der Diebe und Gauner.“ In den Parlamentswahlen am 4. Dezember 2011 erlitt die Regierungspartei schwere Verluste. Auch die Popularitätswerte für Putin waren in dieser Zeit stark zurückgegangen. Entsprechend blieben die für ihn in der Präsidentschaftswahl am 4. März 2012 erzielten Ergebnisse hinter den in der vorangegangenen Wahl erzielten Werten zurück.

Das Bedeutsame an beiden Wahlen waren allerdings nicht nur die Ergebnisse, sondern die Massendemonstrationen gegen Wahlfälschungen und Wahlmanipulationen, die nach beiden Wahlen stattfanden. Diese kamen sowohl für westliche als auch für russische Beobachter ganz unerwartet, denn in früheren Jahren erschienen zu regierungskritischen Demonstrationen lediglich einige Dutzend oder einige Hundert Teilnehmer, denen jeweils

29 Anders Åslund: The Kremlin's New Policy in Its Near Abroad, The Moscow Times, 27.7.2010. eine Armee von Sondereinheiten der Polizei gegenüberstand. Nun aber gingen Zehntausende von Menschen auf die Straße, die ein „Russland ohne Putin“ wollten.

Das andere bedeutsame Phänomen dieser Ereignisse war die Tatsache, dass die Demonstranten zu einem großen Teil aus jungen und aktiven, mit ausländischen und internationalen Organisationen vernetzten Mitgliedern einer im Entstehen begriffenen Zivilgesellschaft bestanden, aus Akademikern, gut ausgebildeten Fachkräften und in der Privatwirtschaft erfolgreichen Unternehmern.

Die Folgerung, welche der Kreml aus der Legitimitätskrise zog, war offensichtlich: Die unter der Ägide Präsident Medwedews angestrebte sozioökonomische Modernisierung Russlands mittels enger Zusammenarbeit mit den USA und der EU und ihren Mitgliedstaaten, nicht zuletzt Deutschlands, hatte offenbar Erwartungen genährt, die für das politische System Russlands gefährlich wurden. Aus Sicht der Moskauer Machtelite war der in Ostmitteleuropa und auf dem Balkan verbreitete Virus der „Farbrevolutionen“ infolge der Einwirkung von westlichen Geheimdiensten und sogenannten Nichtregierungsorganisationen (NGOs) auf Russland übergeschwappt. Dieser Wahrnehmung zufolge musste dieser Entwicklung im Lande selbst und in den Nachbarstaaten energisch Einhalt geboten werden.

Die Maßnahmen, welche diesem Ziel entsprechend getroffen wurden und die bis zum heutigen Tage anhalten, lassen sich nach drei Bereichen unterscheiden: (1) in der Innenpolitik waren dies repressive Maßnahmen zur Abwehr pro-westlicher, demokratischer, liberaler, rechtsstaatlicher und autonomer zivilgesellschaftlicher Strömungen; (2) um die Legitimitätsbasis des Systems Putin zu verbreitern wurde eine national-patriotische anti-westliche Mobilisierung eingeleitet. (3) In der Außenpolitik bemüht sich Russland seither, die Ausstrahlungskraft, den Modellcharakter, die innere Stabilität und die zwischenstaatliche Zusammenarbeit westlicher demokratischer, pluralistischer und rechtsstaatlicher Systeme zu untergraben.

\section{Die Wende zur strategischen Gegnerschaft}

Das erste deutliche Signal für das Ende staatlicher Duldung von Kritik am System Putin wurde am 6. Mai 2012 gegeben. ${ }^{30}$ Auf die Protestkundgebungen an diesem Tag

$30 \mathrm{Zu}$ den innenpolitischen Maßnahmen von Abgrenzung zum Westen und Ausgrenzung ausländischer „Agenten“ und „unerwünschter ausländischer Organisationen im Inland“ siehe Adomeit 2016. 


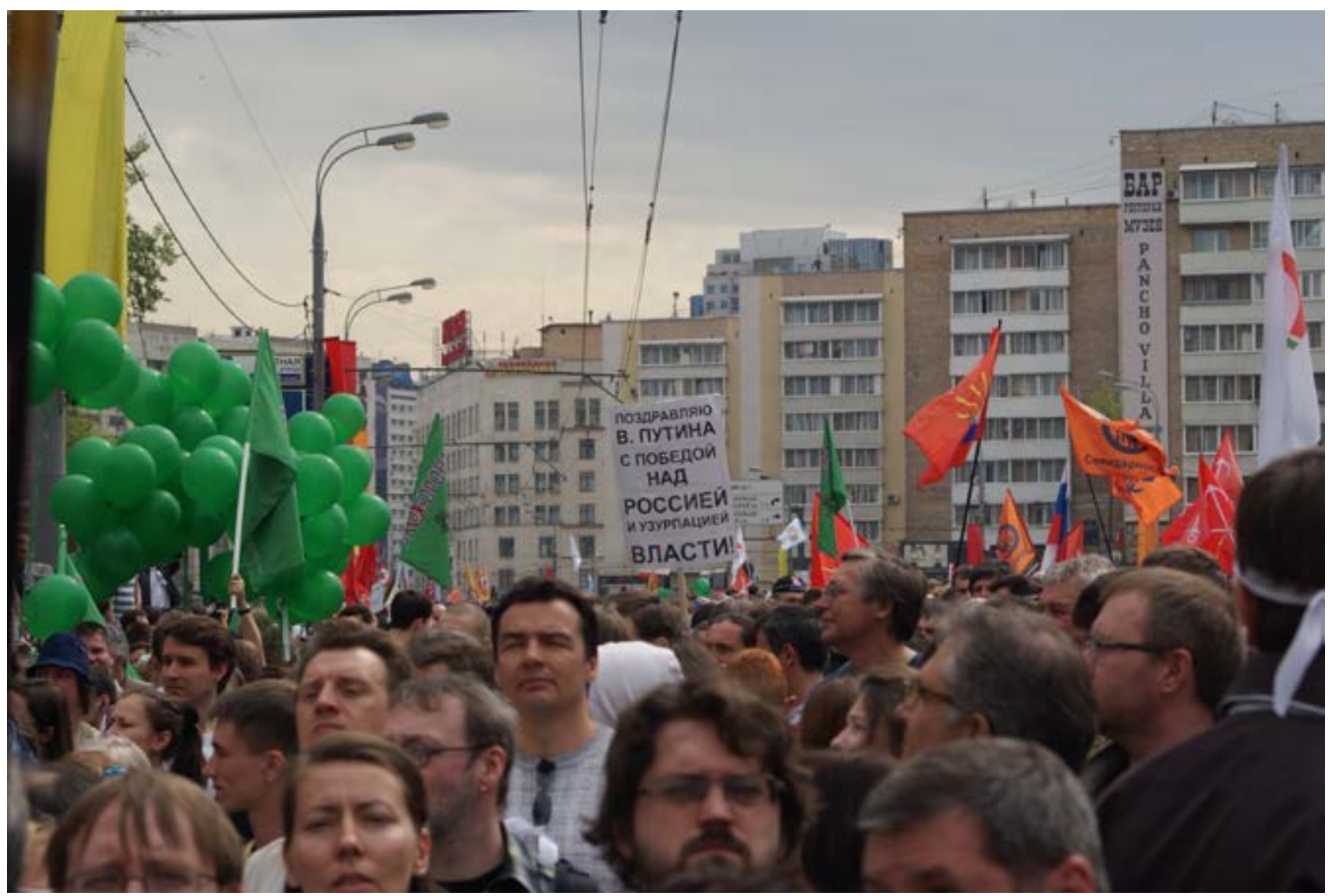

Proteste gegen Putins Amtsantritt als Präsident 2012

gegen die Inauguration Putins für eine dritte Amtszeit als Präsident reagierten die Staatsorgane mit Großaufgeboten von Sicherheitskräften, großer Härte, Verhaftungen und Strafverfahren. An zwei bewilligten Kundgebungen in Moskau nahmen Zehntausende von Menschen teil, die zum Versammlungsort gelangen wollten, dem BolotnajaPlatz in Moskau. Dabei kam es zu Ausschreitungen, die vermutlich von regimetreuen Anhängern bewusst provoziert wurden, um die bis dahin friedliche Protestbewegung zu diskreditieren und den Sondereinheiten der Polizei (OMON), den Vorwand zu liefern, brutal zuzuschlagen. ${ }^{31}$ In der Folge wurden Teilnehmern an den Protestkundgebungen in umstrittenen Verfahren der Prozess gemacht (,Bolotnaja-Prozesse“), bei denen Putin-Gegner zu mehreren Jahren Straflager verurteilt wurden.

31 Einen überzeugender Indiziennachweis für die These, dass das Umschlagen der bis dahin friedlichen Proteste in Gewalt von regimetreuen Anhängern provoziert wurde, liefert Gesine Dornblüth: Konstruierter Anlass für Repressalien gegen Putin-Gegner, Deutschlandfunk.de, 20.4.2013; desgl. Nach dem Urteil im Bolotnaja-Prozess: Hunderte Festnahmen bei Protesten in Moskau, Süddeutsche Zeitung online, 14.4.2014.
Dem folgte eine Vielzahl von Abwehrmaßnahmen gegen angebliche oder tatsächliche westliche Einflussnahmen. So erließ die Duma am 20. Juli 2012 ein Gesetz, demzufolge russische Vereine und Menschenrechtsgruppen unter Androhung schwerer Strafen verpflichtet werden, sich in einem besonderen Register des Justizministeriums als „Agenten des Auslandes“ eintragen zu lassen, sofern sie sich politisch betätigen und Spendengelder aus dem Ausland erhalten. Im September 2012 untersagte das russische Außenministerium praktisch mit sofortiger Wirkung der US Agency for International Development (USAID) jegliche Tätigkeit in Russland.

Am 23. Mai 2015 wurde ein weiteres wichtiges Instrument der Abgrenzung vom Westen und der Diskreditierung von Zusammenarbeit mit westlichen NGOs in Kraft gesetzt - das Gesetz über „unerwünschte Organisationen." Dieses richtet sich zwar gegen ausländische und internationale Nichtregierungsorganisationen, aber aufgrund der Tatsache, dass derartige NGOs in der Regel russische Kooperationspartner und Mitarbeiter haben, auch gegen russische Staatsbürger. In der Begründung des Gesetzes heißt es, in Russland tätige „zerstörerische Organisationen“ müssten gestoppt werden. Sie seien eine 
Bedrohung für die „Werte des russischen Staates“ und sie könnten Umstürze in russischen Nachbarstaaten anzetteln. ${ }^{32}$

Im November 2012 wurde die Gesetzgebung über Hochverrat und Landesverrat erweitert und ihre Anwendung verschärft. Nach der Annexion der Krim und der militärischen Intervention Russlands in der Ostukraine verdreifachte sich die Anzahl der wegen dieses schwerwiegenden Delikts verurteilten Personen und stieg danach weiter an. Zudem verabschiedete die Duma am 24. Juni 2016 ein Anti-Terror-Gesetzespaket, demzufolge Messenger-Dienste verpflichtet werden, Sicherheitsbehörden die Dekodierungsschlüssel für die Nachrichten ihrer Nutzer bereitzustellen. „Unterlassene Meldung einer Straftat“ sowie „Anstiftung, Anwerbung oder anderweitige Heranziehung zur Organisation von Massenunruhen“ fanden Einzug in die Gesetzbücher und wurden mit schweren Strafen belegt.

Die Besorgnis des Kremls über das mögliche Ausbrechen einer „Farbrevolution“ in Russland fand am 3. Juli 2016 einen weiteren Niederschlag in der Schaffung eines neuen föderalen Sicherheitsorgans, der Nationalgarde (Rosgvardia) - die bedeutendste Restrukturierung von Russlands inneren Sicherheitsorganen seit mehr als zehn Jahren. Dazu wurden die Inneren Truppen und die Spezialkräfte der Polizei dem Innenministerium entzogen und direkt dem Präsidenten unterstellt, also eine Art präsidiale Prätorianergarde geschaffen. Das Aufgabengebiet der Truppe schließt den „Schutz der öffentlichen Ordnung“ sowie die Bekämpfung von „Extremismus“ und „Terrorismus“ ein. ${ }^{33}$

Im Zusammenhang mit der Verschärfung der Gesetzgebung und „administrativen“ Maßnahmen gegen ausländische „Agenten“ und „unerwünschte“ ausländische Organisationen leitete der Kreml eine Kampagne mit dem Ziel ein, die westlichen Demokratien zu diskreditieren und zu diffamieren und das eigene Wertesystem als vorbildhaft hinzustellen. So führte Putin in einer Rede 2013 auf dem Waldaj-Diskussionsforum aus, die „euro-atlantischen Staaten“ lehnten ihre christlichen Wurzeln ab, welche die Grundlage der westlichen Zivilisation bildeten. Sie verneinten die „moralischen Grundlagen und jede traditionelle Identität - nationale, religiöse, kulturelle oder sogar geschlechtliche Identitäten und ver-

32 Einzelheiten und Quellennachweise bei Adomeit 2017a.

33 Putin podpisal zakon o vojskach nacional'noj gvardii RF, Rg.ru, 3.7.2016, https://rg.ru/2016/07/03/putin-podpisal-zakon-o-vojskahnacionalnoj-gvardii-rf.html. Einzelheiten des Gesetzes und Analyse bei Klein 2016. suchen, dieses Modell den anderen Ländern weltweit aggressiv aufzuzwingen. ${ }^{\text {"34 }}$

Dem angeblichen Kulturverfall des Westens hat Putin unter anderem das Konzept der Russischen Welt (Russkij mir) entgegengesetzt. ${ }^{35} \mathrm{Zu}$ dieser Welt gehörten nicht nur über 30 Millionen ethnische Russen im Ausland, die russische „Volksgemeinschaft“ (obščina) und „Russischsprachige“ (russkojazyčnye), sondern sie vereine „all diejenigen, die die russische Sprache und Kultur wertschätzen, ganz unabhängig davon, wo sie leben, sei es in Russland oder jenseits seiner Grenzen.“36 Die zivilisatorische Identität basiere auf der „Erhaltung kultureller Dominanz Russlands, die nicht [allein] von den ethnischen Russen bestimmt wird, [sondern von all denjenigen Menschen, die sich dieser Identität] unabhängig von ihrer Nationalität verpflichtet fühlen. “ ${ }^{37}$ Ein weiteres grundlegendes Element dieser Identität sei die „Rechtgläubigkeit“ (pravoslavie) eine Feststellung, die der Russischen Orthodoxen Kirche einen bedeutenden Platz in der Legitimierung des Systems Putins und seiner Außenpolitik zuweist. ${ }^{38}$

Zur Legitimierung der anti-westlichen Orientierung und der Anwendung militärischer Instrumente in der Außenpolitik schmiedet der Kreml auch die Geschichte $\mathrm{zu}$ einer Waffe. ${ }^{39} \mathrm{Er}$ lässt diese in strahlendem Glanz erscheinen. Russland habe immer wieder „glänzende“ militärische Siege über die Invasoren aus dem Westen errungen, seien es die Kreuzritter, die Armee Napoleons oder die Wehrmacht Nazi-Deutschlands. Der „Große Vaterländische Krieg“, also der Zweite Weltkrieg, wird als Paradebeispiel für die aus dem Westen drohenden Gefahren behandelt und gleichzeitig als Beweisstück für Patriotismus und Opferbereitschaft der Bevölkerung und für die Not-

34 Diskussionnyj klub „Valdaj“, Vystuplenie Vladimira Putina, Youtube.com, 19.9.2013, https://www.youtube.com/watch?v=XGCUg 1AEzy4.

35 Zum Konzept der Russischen Welt als Soft-Power Instrument in der Putinschen Außenpolitik siehe Quiring 2017.

36 Putin Ende 2006 auf einem Kongress in St. Petersburg zum Jahr der russischen Sprache, zit. Von Lawrow im Rahmen eines Interviews, Lavrov nazval vnešnopolitičeskim prioritetom Rossii podderžku „Russkogo mira“, Rifinfo.ru, 2.11.2015; http://www.rifinfo.ru/ news/41125.

37 Putin in einem richtungsweisenden Artikel für die Zeitung Nezavisimaja gazeta vom 23.1.2012, publiziert offensichtlich im Zusammenhang mit den Präsidentschaftswahlen am 4.3.2012, Vladimir Putin: Rossija - nacional'nyj vopros, Ng.ru, 23.1.2012, http://www.ng.ru/ politics/2012-01-23/1_national.html.

38 So der russische Außenminister in einem Interview, Lavrov nazval vnešnopolitičeskim prioritetom Rossii podderžku „Russkogo mira“, Rifinfo.ru, 2.11.2015; a.a.O.

39 Zur Geschichte als Instrument der russischen Innen- und Außenpolitik siehe Stewart 2020 und Kolesnikov 2020. 
wendigkeit, stets militärisch gerüstet zu sein, angeführt. Trotz dunkler Seiten sei Stalin als kluger Militärführer und Staatsmann zu werten; der Molotow-Ribbentrop-Pakt sei keineswegs als moralisch verwerflich zu verurteilen, sondern er sei objektiv notwendig gewesen - ein historisch gerechtfertigter Akt der Staatsräson. Die damit verbundenen Schrecken der sowjetischen Besatzung Polens und des Baltikums sowie des Angriffskrieges gegen Finnland im Zeitraum vom September 1939 bis Juni1941 werden ignoriert. Die Wiedererrichtung der Sowjetherrschaft in Polen und im Baltikum nach 1944 wird als „Befreiung“ dargestellt.

Der angeblichen Notwendigkeit, der strategischen Herausforderung durch den Westen zu begegnen, entspricht dann auch die umfassende Modernisierung der Streitkräfte. Grundlage dazu war das schon vor der Wende zur national-patriotischen Mobilisierung Ende Dezember 2010 beschlossene Staatliche Rüstungsprogramm 2011-2020 (GPV-2020), für das Finanzmittel in Höhe von 23 Billionen Rubel (zum damaligen Zeitpunkt 755 Milliarden US-Dollar) bereitgestellt werden sollten. ${ }^{40}$ Das Programm wiederum war Teil einer von Verteidigungsminister Anatolij Serdjukow nach dem Georgien-Krieg in Gang gesetzten Militärreform. ${ }^{41}$ Diese schaffte wesentliche Voraussetzungen für die Anwendung militärischer Macht und der Drohung ihres Einsatzes in der russischen Außenpolitik.

\section{Militärische Macht als außen- politische Waffe}

Die westliche Fixierung seit 2014 auf den „hybriden“ Krieg als neuer Kriegstypus mit entsprechender Streitkräfte- und Rüstungsplanung trübt den Blick dafür, dass die politische und militärische Führung Russlands keineswegs das „traditionelle"Ziel aufgegeben hat, die russischen Streitkräfte auf einen Krieg „großen Maßstabs“, also einen Krieg mit der NATO, vorzubereiten. ${ }^{42}$ Das hat Generalstabschef Gerassimow noch einmal mit aller Deutlichkeit festgestellt: „Die Wahrscheinlichkeit, dass Krisensituationen außer Kontrolle geraten und sich zu einem großen militärischen Konflikt entwickeln, kann nicht ausgeschlossen werden.

40 GPV: Gosudarstvennaja programma vooruženij.

41 Einer Studie von Chatham House zufolge war das Staatliche Rüstungsprogramm GPV-2020 sehr erfolgreich; es wurden „bedeutsame Ergebnisse erzielt: Connolly/Boulège 2018, 7.

42 Begriffe wie Krieg oder militärischer Konflikt „großen Ausmaßes“ (krupnomasštabnaja vojna; voennyj konflikt bol'šogo masštaba) bedeuten im Klartext Krieg mit der Nato.
Gleichzeitig lassen die Tendenzen der Veränderung der Formen des bewaffneten Kampfes den Schluss zu, dass die Kriege der Zukunft in allen Sphären geführt würden. “43

Die Vorbereitungen des Generalstabs auf einen Krieg mit der NATO betreffen sowohl die nuklearstrategischen und die mit taktischen Atomwaffen ausgerüsteten Truppen als auch die konventionellen Streitkräfte. Aus ihren tatsächlichen oder angeblichen Fähigkeiten wird kein Geheimnis gemacht, sondern diese werden gezielt als Drohpotential genutzt. Ein entsprechendes Signal im nuklearstrategischen Bereich sendete der Kreml bereits im Juli 2007 aus, als er die Wiederaufnahme regelmäßiger Patrouillenflüge im internationalen Luftraum vor der amerikanischen Küste mit atomwaffenfähigen strategischen Bombern ankündigte. Ein weiterer Meilenstein war Putins computergestützte Videopräsentation vor rund 1.000 Spitzenpolitikern, Regierungsvertretern und „einfachen Bürgern“ am 1. März 2018, auf der er neue „unbesiegbare Atomwaffen“ vorstellte, einschließlich einer Interkontinentalrakete RS-28 Sarmat, die über 10-24 Sprengköpfe verfüge und mit einer Reichweite von über 11.000 Kilometern die USA über den Nord- oder Südpol ansteuern und durch ihre Mehrfachsprengköpfe die US-Raketenabwehr überwinden könne; eines atomgetriebenen Marschflugkörpers mit interkontinentaler Reichweite (Burewestnik) und großer Manövrierbarkeit; und „Hyperschallwaffen“ wie ein von Flugzeugen abzufeuernder konventioneller Überschall-Marschflugkörper namens Kinschal.

Bei den Nuklearwaffen mittlerer und kürzerer Reichweite, bei denen Russland eine große numerische Überlegenheit besitzt, fällt vor allem die Stationierung von drei mit dem neuen Mittelstrecken-Raketensystem 9M729 ausgerüsteten Bataillonen im europäischen Teil Russlands ins Gewicht. Die Entwicklung und Stationierung des 9M729 Marschflugkörpers war der Grund für den Austritt der USA aus dem INF-Vertrag.

$\mathrm{Zu}$ seinen nuklearen Drohgebärden gegenüber den europäischen Nato-Staaten hat der Kreml auch das Iskander-M-System herangezogen, das mit der ballistischen Kurzstreckenrakete $9 M 723$ bestückt ist und mit einer Reichweite von 500 Kilometern Warschau, Berlin oder Kopenhagen erreichen kann. Die Raketen waren zuerst mehrere Male auf Militärparaden und bei Militärmanövern

43 Auf einem Treffen mit den in Moskau akkreditierten ausländischen Militärattachés: Ministerstvo oborony RF otkryto k ravnopravnomu dialogu po voprosam obespečenija voennoj bezopasnosti: Krasnaja zvezda, Redstar.ru, 18.12.2019, http://redstar.ru/ ministerstvo-oborony-rf-otkryto-k-ravnopravnomu-dialogu-po-voprosam-obespecheniya-voennoj-bezopasnosti/. Gerasimow bezieht sich hier auf den „Weltraum als fünftes Operationsfeld“. 
in der Exklave Kaliningrad zu sehen und wurden ab 2018 dort dauerhaft stationiert. Wohl gerade nicht zur Beruhigung, sondern zur gezielten Beunruhigung der europäischen Nachbarn sagte ein russischer Militärsprecher, dass die Iskander auf dem Kaliningrader Gebiet nicht übungshalber abgefeuert würden, „um unsere Nachbarn in Polen und Litauen nicht in Schockstarre zu versetzen“. ${ }^{44}$

Der Generalstab arbeitet weiter an der Ausformung seiner langfristigen Strategie, die Bereitschaft der russischen Streitkräfte für eine umfassende Konfrontation mit der NATO zu erhöhen und regionale Überlegenheit auch bei den konventionellen Waffen zu erringen. ${ }^{45}$ Beispiele hierfür sind die Aufstellung eines neuen Panzerregiments im vorletzten Jahr und eine im Kaliningrader Gebiet geschaffene motorisierte Schützendivision im Jahr 2020 die sechste, die in den letzten sieben Jahren in westlicher Richtung gegründet wurde. Die Luftstreitkräfte sollen reformiert und modernisiert werden, sodass diese in Zukunft Aufgaben über das gesamte Spektrum militärischer Operationen hinweg ausführen können, vom lokalen Konflikt bis zur groß angelegten konventionellen Kriegsführung. Dazu sollen auch sogenannte luftbewegliche Brigaden dienen, die von neuen speziellen Kampfhubschraubern begleitet, autonom agieren und die Gegner mit präventiven Schlägen überraschend attackieren können. Im Rahmen der Reform plant der Generalstab auch, die bestehenden Einheiten der Luftstreitkräfte durch vier Luftmobilbrigaden $\mathrm{zu}$ ersetzen - eine für jede strategische Richtung, wobei die 31. Luftmobilbrigade in Uljanowsk die strategische Ausrichtung des Westens abdecken soll.

Seit 2018 wurden luftmobile Einheiten in allen wichtigen Übungen getestet (Wostok 2018, Zentr 2019 und Kawkas 2020). Auch im Westlichen Militärbezirk hat das Verteidigungsministerium Militärmanöver fortgesetzt. Ende August 2020 führten die 6. russische kombinierte Waffenarmee und die baltische Flotte ihre erste gemeinsame Übung im Finnischen Meerbusen durch, die eine amphibische Landung auf der Insel Hogland beinhaltete. Russische Militärflugzeuge fliegen auch weiterhin mit ausgeschalteten Transpondern, und die Aufklärungsflüge haben nicht aufgehört. Dieses Jahr wird eine weitere wichtige Übung in europäischer Richtung durchgeführt, Zapad2021. Daran werden vermutlich wieder Zehntausende von Truppen entlang der Grenzen der baltischen Staaten, und Polens teilnehmen, traditionell eine Übung, die einen um-

44 Russland lässt „Iskander“-Raketen in Kaliningrad auffahren, Spiegel online, 4.5.2018.

45 Die nachfolgende Beschreibung der Anstrengungen, die Russland im Bereich der konventionellen Streitkräfte und Waffen unternimmt, folgt dem Estonian Foreign Intelligence Service Report 2021, 45-51. fassenden Konflikt mit der NATO in Europa zum Gegenstand hat.

Wiederum aller Wahrscheinlichkeit nach nicht als Beruhigung, sondern als Warnung an alle diejenigen, die sich Moskaus Willen nicht beugen wollen, hat Putin dem Präsidenten eines europäischen Landes lapidar zu verstehen gegeben: „Wenn ich wollte, könnten russische Truppen in zwei Tagen nicht nur in Kiew, sondern auch in Riga, Vilnius, Tallinn, Warschau oder Bukarest sein.“46

Als Druck und Drohung waren sicher auch die russischen Truppenbewegungen im Frühjahr 2021 zu verstehen: Im Februar hielt das russische Verteidigungsministerium Manöver auf der Krim ab, an denen 3.000 Fallschirmjäger und amphibische Einheiten teilnahmen. Dem Ministerium zufolge sei Ziel der Übung das „Ergreifen von Objekten der Feinde mit anschließender Verteidigung bis zur Vereinigung [der Vorauskräfte] mit der Hauptstreitmacht“ gewesen. ${ }^{47}$ Im April gab Putins Sprecher Dmitri Peskow im Zusammenhang mit Manövern russischer Militäreinheiten auf russischem Gebiet in Grenznähe sowie vermehrten Gefechten in der umkämpften Ostukraine Kiew die Schuld an den Spannungen, denn die ukrainische Armee habe „zahlreiche Provokationen“ verübt. Daraufhin warnte er, dass jeder Einsatz von NATO-Truppen „zweifellos zu weiteren Spannungen führen“ würde, denn die russische Seite müsste „zusätzliche Maßnahmen ergreifen, um ihre Sicherheit zu gewährleisten“. ${ }^{48}$ Und Außenminister Lawrow zitierte ein Wort Putins, wonach jeder, der „versucht, einen neuen Krieg im Donbass zu entfesseln, die Ukraine zerstören wird“. ${ }^{49}$

46 Putin am 12. September 2014 in einem Gespräch mit dem ukrainischen Präsidenten Präsident Poroschenko in Kiew. Nach einem Bericht der Süddeutschen Zeitung, die sich auf eine ihr vorliegende Gesprächszusammenfassung des Auswärtigen Dienstes der EU über ein Treffen des EU-Kommissionspräsidenten José Manuel Barroso beruft; siehe Daniel Brössler: Putin soll Europa massiv gedroht haben, Süddeutsche Zeitung online 18.9.2014. Anfang des Monats war bereits berichtet worden, Putin habe in einem Telefonat mit Barroso gesagt: „Wenn ich wollte, könnte ich Kiew binnen zwei Wochen einnehmen.“ Der Kreml hatte diese Drohung daraufhin nicht geleugnet, sondern lediglich kritisiert, dass Barroso den Inhalt eines privaten Telefonats publik gemacht habe.

47 Andrew Kramer: After Months Of Inactivity, Fight Restarts In Ukraine, New York Times, 30.3.2021, A 12. Mit den „Objekten“ konnte oder sollte wohl die „Ergreifung“von Wasserkanälen auf der der Krim gegenüberliegenden ukrainischen Seite gemeint gewesen sein, um die Wasserversorgung der Halbinsel zu sichern.

48 Peskov otvetil na plany SŠA zaščitit’ Ukrainy ot „rossijskoj aggressii“. Rbc.ru; https://www.rbc.ru/politics/02/04/2021/6066ec3b9 a7947547271f9e7.

49 Interv'ju Ministra inostrannych del Rossijskoj Federacii S.B. Lavrova programme „Bol'šaja igra“ na „Pervom kanale“, Moskva, 1 aprelja 2021 goda, Mid.ru, 1.4.2021, https://www.mid.ru/meropriyatiya_s_ 


\section{Der postsowjetische Raum als Austragungsraum der Gegnerschaft}

Der oben beschriebene Begriff der Russischen Welt und seine Inhalte sind von zentraler Bedeutung für die Systemkonkurrenz zwischen dem Autoritarismus Putinscher Prägung und westlichen Demokratien. Einen fairen Wettbewerb schränkt Moskau jedoch durch seinen Anspruch auf den postsowjetischen Raum als russische Einflusssphäre ein. Eine der konkreten Ausprägungen dafür sind Abgrenzung und Abwehr politischer und wirtschaftlicher Aktivitäten der Europäischen Union im postsowjetischen Raum.

Schon kurz nach dem Start der EU-Initiative der Östlichen-Partnerschaft hatte sich Außenminister Lawrow beklagt: „Wir werden beschuldigt, über Einflusssphären zu verfügen. Aber was ist die Östliche Partnerschaft anderes als ein Bemühen, die europäische Einflusssphäre auszudehnen?" ${ }^{50}$ In seiner damaliger Eigenschaft als Präsident stellte Medwedew lapidar fest: „Wenn die Ukraine den Weg der europäischen Integration geht, wird es für das Land schwieriger, sich in den Gemeinsamen Wirtschaftsraum und die Zollunion zu integrieren. Man kann nicht gleichzeitig auf zwei Stühlen sitzen. “51 Noch schärfer hat dies Sergej Glasew, Putins Berater in Fragen der eurasischen Wirtschaftsintegration, formuliert: „Die Zollgrenze [Russland, Belarus und Kasachstan] - das ist eine Linie von Barrikaden. Wenn Sie auf der einen Seite der Barrikaden, im Eurasischen Wirtschaftsraum sind, bedeutet dies, dass Sie alle ihre Ressourcen bekommen. Stehen Sie auf der anderen Seite der Barrikaden, dann erhalten Sie all die Segnungen der modernen Marktbeziehungen. ${ }^{\text {"52 }}$

Aus der Sicht des Kremls waren die zu ziehenden Konsequenzen eindeutig: Eine de facto Integration der für den Erfolg der Eurasischen Wirtschaftsunion wichtigen Ukraine in die EU mithilfe des im November 2013 unterschriftenreifen Assoziierungsabkommens musste mit

uchastiem_ministra/-/asset_publisher/xK1BhB2bUjd3/content/ id/4662534.

50 EU Expanding its „Sphere of Influence“, Russia Says, Euobserver. com, 21.3.2009, https://euobserver.com/foreign/27827.

51 Auf einer Pressekonferenz im Innovationszentrum Skolkovo, RIA Novosti, Rian.ru, 18.5.2011, http://en.rian.ru/world/20110518/16409 2502 [Hervorhebung nicht im Original].

52 Sovetnik Putina porosil Ukrainu vybrat', po kakuyu stroronu barrikad žit', Rus.lb.ua, 26.4.2013, https://rus.lb.ua/news/2013/04/26/ 198277_sovetnik_putina_poprosil_ukrainu.html [Hervorhebung nicht im Original]. allen Mitteln verhindert werden. Mit der Weigerung des ukrainischen Präsidenten Viktor Janukowitsch, das Abkommen zu unterschreiben, schien dieses Ziel gesichert zu sein. Die Maidan-Farbrevolution machte diese Erwartung allerdings zunichte und setzte einen Prozess in Gang, der über die Annexion der Krim und die Militärintervention in der Ostukraine zu westlichen Sanktionen und damit zu einer weiteren Ausprägung der Gegnerschaft zwischen Russland und dem Westen führte.

Dies gilt auch für die Reaktionen des Kremls auf die arabische Variante der ostmitteleuropäischen „Farbrevolutionen“, den „Arabischen Frühling“.

\section{Libyen, Syrien und der Nahe Osten als Austragungsraum der Gegnerschaft}

In den ersten Monaten nach dem Beginn der Massendemonstrationen gegen das Regime Muammar al-Gaddafis in Libyen am 15. Februar 2011 reagierten der Kreml und die staatlichen Medien im Wesentlichen noch im Einklang mit westlichen Analysen. So stellte beispielsweise Außenminister Lawrow fest: „Die treibende Kraft hinter den Ereignissen in Libyen, die wir [auch] in Ägypten und anderen Ländern beobachtet haben, [ist] hauptsächlich die gebildete Jugend." ${ }^{53}$ Dem stimmte der stellvertretender Außenminister Michail Bogdanow mit den Worten zu: „Die Ursachen der gegenwärtigen Turbulenzen in den arabischen Ländern liegen [...] sowohl im sozioökonomischen als auch im politischen Bereich. [...] Die Bewegungslosigkeit von Führern und politischen Eliten, das geringe Maß an sozialer Mobilität, der späte Beginn beziehungsweise sogar das Fehlen dringender Reformen, die hohe Arbeitslosigkeit, Korruption und andere gesellschaftliche Krankheiten - all diese internen Konfliktfaktoren haben sich über viele Jahre angesammelt und sind Anfang dieses Jahres explodiert.“54

53 Lawrow, Interview mit Fernsehmoderator Vladimir Solov'ev, 13. März 2011: Interv'ju Ministra inostrannych del Rossii S.V. Lavrova [...] V. Solov'evu, Mid.ru, 13.3.2011, https://www.mid.ru/foreign_ policy/news/-/asset_publisher/cKNonkJE02Bw/content/id/215526; zit bei Issaev 2021, 2.

54 Bogdanow, Interfax-Interview, 5. Juli 2011: Interv'ju zamestitelja Ministra inostrannych del Rossii M. Bogdanova agenstvu „Interfaks“, Mid.ru, 5.7.2011, https://www.mid.ru/web/guest/foreign_policy/ news/-/asset_publisher/cKNonkJE02Bw/content/id/200982, zit. ebd. 
Im Einklang mit derartigen Wahrnehmungen des „Arabische Frühlings“ hatte Präsident Medwedew Russlands Enthaltung bei der UN-Resolution 1973 vom 17. März 2011 verfügt, welche die Mitgliedsstaaten ermächtigte, „alle notwendigen Maßnahmen“, also auch militärische, zu ergreifen, um von Angriffen bedrohte Zivilpersonen und von der Zivilbevölkerung bewohnte Gebiete zu schützen. Diese Entscheidung führte zu einer bemerkenswerten und präzedenzlosen, öffentlich ausgetragenen Auseinandersetzung zwischen Präsident und Premier. Am 21. März kritisierte Putin die Resolution als „mangelhaft und fehlerbehaftet“, die „Leichtigkeit“, mit der die Entscheidung zum Einsatz von Gewalt getroffen worden sei und als eine Art „mittelalterlicher Aufruf zum Kreuzzug“. Nur Stunden später bezeichnete Medwedew Putins Aussage als „inakzeptabel“ und konterte: „Russland hat [das Vetorecht] aus dem Grund nicht ausgeübt [weil] ich die Resolution nicht für falsch halte. Darüber hinaus glaube ich, dass diese Entscheidung unser Verständnis der Entwicklungen in Libyen widerspiegelt. Es ist absolut unentschuldbar, Ausdrücke zu verwenden, die tatsächlich zu einem Zusammenprall der Zivilisationen führen könnten - wie ,Kreuzzüge“ und so weiter." ${ }^{\text {"55 }}$

Allerdings setzte sich Putin mit seiner Sicht der Dinge durch. Er machte den Westen, insbesondere die USA, für die Unruhen verantwortlich, die wiederum - auch mithilfe militärischer Mittel - dazu dienen sollten, Regimewechsel herbeizuführen. Nach der Bombardierung Jugoslawiens und den Militäroperationen in Afghanistan sowie im Irak sei ,jetzt Libyen an der Reihe“ ${ }^{56}$ Im Kern nahm Putin damit die von Generalstabschef Gerassimow in seiner Rede im Februar 2013 gemachten Ausführungen über „neue“ westliche „nicht-lineare“ Kriegsführung vorweg. Ihm zufolge bestätigten „die Erfahrungen mit militärischen Konflikten, einschließlich solcher im Zusammenhang mit den sogenannten Farbrevolutionen in Nordafrika und im Nahen Osten, dass ein völlig blühender Staat in wenigen Monaten und sogar Tagen in eine Arena heftiger bewaffneter Konflikte verwandelt und Opfer ausländischer Interventionen wird sowie in einem Netz aus Chaos, humanitärer Katastrophe und Bürgerkrieg versinken kann. Natürlich ist es am einfachsten zu sagen, dass die Ereignisse des Ara-

55 Predsedatel' Pravitel'stva Rossii V.V. Putin [...] prokommentiroval situaciju vokrug Livii, 21-ogo Marta 2011, Archive.government. $r u$, http://archive.government.ru/special/docs/14542/. Zu den unterschiedlichen Positionen Medwedews und Putins siehe Krestonoscy Medvedev i Putin kardinal'no razošlis' po livijskomu voprosy, Lenta. ru, 22.3.2011, https://lenta.ru/articles/2011/03/22/rulibya/.

56 Ebd. bischen Frühlings kein Krieg sind und wir - Militärs daher nichts daraus lernen können. Aber vielleicht ist das Gegenteil der Fall, dass genau diese Ereignisse typisch für die Kriegsführung im 21. Jahrhundert sind.“57

Syrien findet zwar bei Gerassimow keine ausdrückliche Erwähnung, die NATO-Intervention und der Regimewechsel - der Sturz Gaddafis - in Libyen sind aber für das Verständnis der russischen Unterstützung für das Regime Baschar al-Assads und die „Rückkehr“ Russlands in den Nahen und Mittleren Osten von prinzipieller Bedeutung. ${ }^{58}$ Schon im Mai 2011 bezeichnete Lawrow jegliche (westliche) „Versuche, die libyschen Erfahrungen in anderen Ländern und Regionen wie Syrien, Jemen oder Bahrain zu

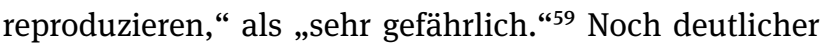
wurde der damalige russische Generalstabschef General Nikolaj Makarow. Nach dem Sturz von Gaddafi behauptete er unverblümt, dass „die Führer einiger Länder“ mithilfe der Techniken der „Farbrevolutionen“ ihnen unbequeme politische Regime (auch in Syrien, Ägypten, Tunesien und Jemen) beseitigen wollen, um ihre strategischen Interessen durchzusetzen. Er befürchtete, dass die gleichen Techniken auf Russland und seine Nachbarn Anwendung finden könnten. ${ }^{60}$

Die innenpolitischen Determinanten - speziell der Wandel vom Medwedewschen Ansatz sozioökonomischer Modernisierung zu Putins national-patriotischer Mobilisierung und Militarisierung der Gesellschaft - liefern einen wesentlichen Teil der Erklärung dafür, warum Moskau seine politische Unterstützung, Waffenlieferungen und die Entsendung von Militärberatern im September 2015 zum direkten militärischen Eingreifen mit eigenen Truppen und dem Auf- und Ausbau von Militärbasen in Syrien ausweitete. Der enorme Popularitätszuwachs mit Spitzenratings für Putin nach der Annexion der Krim und der Militärintervention in der Ostukraine nahm der Kritik an seinem militärischen Engagement in Syrien jegliche Spitze. Die systembedingte Gegnerschaft zum Westen verbreiterte sich damit auch zur geopolitischen Gegnerschaft im Nahen und Mittleren Osten.

57 Gerasimov 2013 [Hervorhebung nicht im Original]. Zur russischen Sicht der „Farbrevolutionen“ als westliche Kriegsführung siehe Bilban/Gringinger 2019.

58 Grundlegend für diesen Zusammenhang ist Allison 2013.

59 Sergej Lawrow: Stenogramma interv'ju Ministra inostrannych del Rossii S.B. Lavrova rossijskim SMI po itogam učastija v zasedanii Arktičeskogo soveta 12 maja 2001 goda, Mid.ru, 13.5.2011, https:// www.mid.ru/sovet-gosudarstv-barenceva/evroarkticeskogo-regionasber-/-/asset_publisher/0vP3hQoCPRg5/content/id/207142.

60 General Makarow in einem Bericht an das Russische Bürgerforum, 18. November 2011, zit. bei Allison 2013, S. 817. 
Der Zusammenfluss geopolitischer und systemspezifisch-ideologischer Aspekte der russischen Gegnerschaft zum Westen zeigt sich auch bei der Entwicklung der russisch-chinesischen Beziehungen.

\section{Die „umfassende strategische Partnerschaft" mit China}

Eine Interpretation der von der Moskauer Machtelite in die Wege geleiteten Wende zu feindseliger Einstellung und Gegnerschaft zum Westen stützt sich auf das Narrativ, dass sich Russland gegen westliche Sicherheitsbedrohungen und insbesondere gegen die NATO-Osterweiterung „wehren“ musste. Teil dieser Erzählung über den prinzipiell „reaktiven“ und „defensiven“ Charakter der russischen Außen- und Sicherheitspolitik ist die Behauptung, wonach die vom Westen gegen Russland verfügten Sanktionen nicht nur nichts bewirkt hätten, sondern „kontraproduktiv“ seien. Sie hätten lediglich dazu geführt, dass sich Russland von Europa abgewandt und sich China zugewandt hätte.

Diese These von Russlands „Wende nach Osten“ in seiner Außen- und Wirtschaftspolitik (pivot to the east) hat der Kreml lautstark unterstützt. Allerdings ist diese Sicht der Dinge schief. Übereinstimmende Interessen und Wahrnehmungen der Machtelite in Moskau und Beijing waren bereits gegen Ende des letzten Millenniums erkennbar. Im September 1995 hatte die NATO Grundprinzipien für die Aufnahme osteuropäischer Länder als Mitglieder in das Militärbündnis angenommen, ${ }^{61}$ was lautstarke Proteste in Russland auslöste und dazu beitrug, dass der pro-westliche Außenminister Andrei Kosyrew im Januar 1996 durch Ewgeni Primakow, Chef des Auslandsgeheimdienstes SVR, ersetzt wurde. Er regte Präsident Boris Jelzin für seinen für April 1996 anberaumten Besuch in Beijing dazu an, Präsident Jiang Zemin vorzuschlagen, den seit 1994 gebrauchten Begriff der „konstruktiven Partnerschaft“ für die russisch-chinesischen Beziehungen zur ,strategischen Partnerschaft" zu erhöhen. ${ }^{62}$

Zeitgleich entwickelten sich die Beziehungen zwischen China und den USA zu einer Krise. Diese erreichte ihren Höhepunkt im Dezember 1995, als Washington in der größten US-Machtdemonstration in Asien seit dem Vietnamkrieg auf Chinas Raketentests in der Nähe von Taiwan

61 NATO Study on Enlargement, 28.9.1995, https://www.nato.int/ cps/en/natohq/official_texts_24733.htm.

62 Wacker 1996, sowie Gregory Shtraks: Sino-Russian Relations and the Lessons of 1996, The Diplomat Online, 13.4.2015. damit reagierte, eine vom Flugzeugträger USS Nimitz angeführte Carrier Task Force durch die Taiwan-Meerenge zu schicken. Zudem einigten sich die Vereinigten Staaten und Japan Anfang April 1996 auf mehrere Maßnahmen zur Stärkung ihres Sicherheitsbündnisses. In demselben Monat fand das Gipfeltreffen zwischen Jelzin und Jiang Zemin statt. Vierzehn Abkommen wurden unterzeichnet, und die beiden Mächte erklärten ihre Absicht, eine "gleichberechtigte, vertrauensvolle Partnerschaft zu entwickeln, die auf strategische Zusammenarbeit im 21. Jahrhundert abzielt“. Diese Formulierung wurde ein Jahr später auf dem Gipfeltreffen in Moskau mit der „RussischChinesischen Gemeinsamen Erklärung zur Multipolarität und zur Bildung einer neuen globalen Ordnung“ weiter vorangetrieben. ${ }^{63}$

Kernpunkte dieses Rückblicks auf die Genesis der russisch-chinesischen „strategischen Partnerschaft“ sind erstens - ganz im Gegensatz zu der 1999 verkündeten EURussland „strategischen Partnerschaft“ - ihre tatsächliche strategische Qualität und zweitens, ihre Stoßrichtung gegen die Vereinigten Staaten.

De facto haben sich die beiden Mächte gegenseitig zugesichert, die jeweils von ihnen beanspruchten Einflusssphären - Russland in Europa und China in Asien - zu respektieren und diese gegen US-amerikanische „Einmischungen" und Einflussnahme abzusichern. Derartigen $\mathrm{Zu}$ - und Absicherungen ist durch gemeinsame Übungen der Seestreitkräfte Nachdruck verliehen worden. Seit April 2012 haben die chinesische Marine Manöver im Gelben, Ost- und Südchinesischen Meer mit russischer Beteiligung und Russland im Japanischen, Ochotskischen Meer und in der Ostsee mit chinesischer Beteiligung abgehalten. ${ }^{64}$ Am 22. Dezember 2020 absolvierten sechs strategische Bomber - vier chinesische und zwei russische - eine gemeinsame Patrouillenmission über das Ostchinesische und das Japanische Meer. ${ }^{65}$ China nahm 2018 auch an dem in Russland abgehaltenen „Wostok“ (Osten) Großmanöver mit rund 3.000 chinesischen Truppen, Panzern, Luftverteidigungskräften und Jagdbombern teil. ${ }^{66}$ Und vermutlich werden sich chinesische Truppen auch zum ersten Mal an dem für 2021 geplanten Großmanöver „Zapad“ (Westen)

\section{Ebd.}

64 Auflistung und Analyse russisch-chinesischer Marinemanöver bei Paul 2019.

65 Vgl. William J. Middendorf: China and Russia: Two Big Threats the U.S. Military Can't Ignore, Nationalinterest.org, 1. Februar, https:// nationalinterest.org/blog/buzz/china-and-russia-two-big-threats-usmilitary-cant-ignore-177394. Die erste Patrouille dieser Art fand im Juli 2019 statt.

66 Zur russisch-chinesischen militärischen Zusammenarbeit siehe Gressel 2018, Paul 2019 und Gorenburg 2020. 
beteiligen, das immer mit zehntausenden von Soldaten an der NATO-Grenze abgehalten wird. ${ }^{67}$

Das heißt nicht automatisch, dass die Streitkräfte das jeweils andere Land im Konfliktfall auch militärisch unterstützen werden. Es bedeutet auch nicht, dass Moskau und Beijing zwangsläufig zu Verbündeten werden. Entsprechend hat Putin auf die Frage, ob Russland ein Militärbündnis mit China schließen wolle, geantwortet, dass „wir uns dieses Ziel bisher nicht gesetzt haben, es im Prinzip aber auch nicht ausschließen“". ${ }^{68}$ Die wachsende streitkräfteübergreifende Kooperation hat allerdings beträchtliche Auswirkungen auf die USA und die NATO-Staaten, denn sowohl Russland als auch China setzen hauptsächlich militärische Instrumente in Verbindung mit politischer Kriegsführung ein, um Kräftegleichgewichte und Strukturen der internationalen Politik zu ihren Gunsten zu verändern. Dies gilt nicht nur für den indopazifischen Raum, sondern auch für den Atlantik bis in den hohen Norden und für die Arktis.

Die Herrschaftssysteme beider Staaten, die durch Autoritarismus, Kollektivismus, Militarismus und Nationalpatriotismus in Verbindung mit der Anwendung immer raffinierterer und tiefer greifender Kontroll- und Überwachungsmitteln der Gesellschaft geprägt sind, ähneln sich zwar, aber die von den Machteliten wahrgenommenen Gefahren und Bedrohungen für die „Sicherheit“ des politischen Systems unterscheiden sich voneinander. Diese werden im Gegensatz zur chinesischen Führung von der Moskauer Machtelite als existenziell bedrohlich wahrgenommen. Um derartigen Gefahren zu begegnen, hat der Kreml, wie oben dargestellt, umfangreiche Abwehrmaßnahmen ergriffen. Der Maxime vom „Angriff ist die beste Verteidigung “ entsprechend, ist der Kreml aber auch zur Anwendung offensiver Maßnahmen übergegangen. Dazu gehört auch das Bemühen, die Ausstrahlungskraft westlicher liberaler und demokratischer Systeme mithilfe von Desinformations- und Destabilisierungskampagnen $\mathrm{zu}$ schwächen oder zu beseitigen.

\section{Desinformation und Destabilisierung}

Eine der probaten Möglichkeiten, um die Legitimität des eigenen Systems und seine moralische Überlegenheit hervorzuheben, sind Anstrengungen, konkurrierende oder

67 Estonian Foreign Intelligence Service Report 2021, 48. 68 Videokonferenz des Valdaj-Clubs am 22.10.2020, http://kremlin. $\mathrm{ru} /$ events/president/news/64261 [Hervorhebung nicht im Original]. gegnerische Systeme zu verunglimpfen. Gerade unter Bedingungen offensichtlicher Defizite des eigenen Systems greifen seine Manager gerne zu derartigen Ansätzen. Soweit diese bereit sind, eigene Schwachstellen und sogar schwere Verbrechen einzuräumen, wird gleich auf den Gegner verwiesen, bei dem alles „noch viel schlimmer“ sei. ${ }^{69}$ Entsprechend bemühen sich die russischen Staatsmedien und sozialen Medien im öffentlichen Raum und verdeckt mithilfe der Geheimdienste und Troll-Fabriken, die politische und gesellschaftliche Wirklichkeit in den euroatlantischen Staaten an derartige Behauptungen anzupassen.

Auf die wachsende Bedeutung derartiger politischer Waffen hatte Generalstabschef Waleri Gerassimow im Januar 2013 in seinen Ausführungen über den „neuen“ Kriegstypus des 21. Jahrhunderts hingewiesen. Nichtmilitärische Mittel, argumentierte er, seien „in einer Reihe von Fällen wesentlich effektiver als militärische Mittel. Sie werden durch verdeckte militärische Maßnahmen ergänzt, unter anderem durch Maßnahmen der nachrichtendienstlichen Abwehr, Spezialkräfteoperationen und die Nutzung des Protestpotenzials der Bevölkerung “ ${ }^{70}$

Um die Arbeit der russischen Staatsmedien „effektiver“ zu organisieren, wurde die Nachrichtenagentur RIA Nowost - die größte und modernste Agentur Russlands mit dem Auslandssender Golos Rossii (Stimme Russlands) zur internationalen Informationsagentur Rossija Segodnja (Russland heute) fusioniert und die neue Mediengruppe in die „Liste der strategischen Unternehmen Russlands“ aufgenommen, die eine besondere Bedeutung für die Verteidigungsfähigkeit und Sicherheit des Landes haben. ${ }^{71}$

Am überzeugendsten dokumentiert sind die Anstrengungen verdeckt und öffentlich operierender staatlicher russischer Stellen zur Beeinflussung der US-amerikanischen Wahlen 2016 zugunsten Trumps. Die umfassendste Beschreibung und Analyse dieses Bemühens findet sich in einem Bericht, der von allen 17 mit Aufklärung befassten staatlichen Organisationen (intelligence agencies) der USA gemeinsam getragen wurde. Dort ist von einer breit gefä-

69 Eines der vielen Beispiele dafür ist Putins Erinnerung an den 70. Jahrestag der Schauprozesse und des stalinistischen Terrors - ein für den Kreml-Chef aufgrund seiner KGB-Vergangenheit sensibles Thema. Niemand sollte versuchen, Russland wegen der großen Säuberungen von 1937 schuldig fühlen zu lassen, meinte er. Der Terror sei vielleicht eine der berüchtigtsten Episoden der Stalin-Ära, aber „in anderen Ländern sind noch schlimmere Dinge passiert“ [Hervorhebung nicht im Original].

70 Gerasimov 2013.

71 „Rossija Segodnja“ vošla v spisok strategičeskich predprijatij RF, Bfm.ru, 6.3.2014, http://www.bfm.ru/news/249756; zu einer Analyse der staatlichen Medienpolitik vgl. Spahn 2021. 


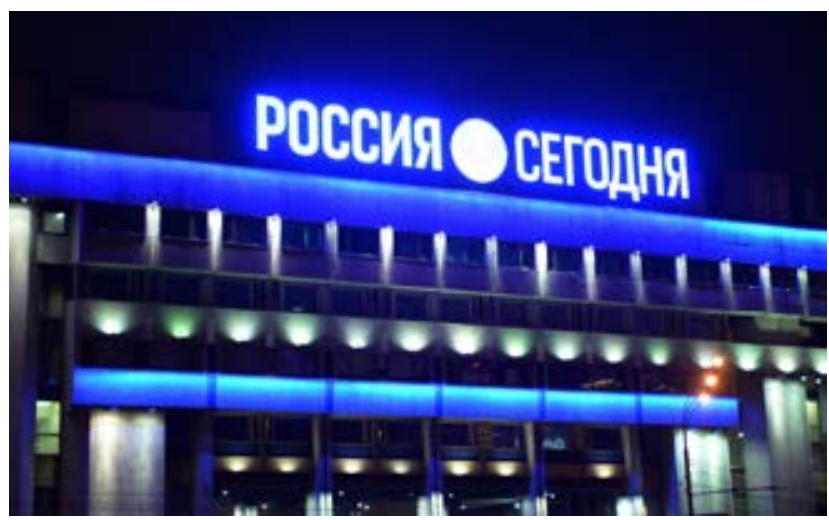

Das Hauptquartier von Rossija Segodnaja im Jahr 2017

cherten Kampagne die Rede, die zum Ziel gehabt habe, „das Vertrauen der Öffentlichkeit in den demokratischen Prozess der USA zu untergraben, die [ehemalige] Außenministerin [Hillary] Clinton zu verunglimpfen, an ihrer Befähigung [Zweifel zu säen] und ihrer Präsidentschaftskandidatur zu schaden“. ${ }^{72}$ Mit anderen Worten: Der Kreml ging - zu Recht, wie sich herausstellte - davon aus, dass ihm eine Trump-Regierung weit mehr nützen würde, als eine von Clinton geführte. Im Rahmen der Wahlen im November 2020 wiederholten sich die Einmischungsversuche Russlands. ${ }^{73}$

Von erheblicher Bedeutung für die Bewertung des russischen Informationskriegs ist allerdings die Tatsache, dass die Kampagne weit über das enge Ziel von Wahlbeeinflussung hinausging und von dem Bemühen getragen wurde, innenpolitische und gesellschaftliche Konflikte zu schüren und Politik und Gesellschaft zu polarisieren. So sind beispielsweise unterschiedliche Forschungsprojekte über die US-Wahlen $2016 \mathrm{zu}$ dem Ergebnis gekommen, dass in den sozialen Netzwerken gezielt Afroamerikaner ins Visier genommen wurden, um sie vom Wählen abzuhalten und ihren Glauben an das politische System zu erschüttern. ${ }^{74}$

Der von Putin seit Beginn seiner dritten Amtszeit gegen den Westen gerichtete Informationskrieg und das Bemühen, innenpolitische und gesellschaftliche Konflikte $\mathrm{zu}$ schüren, wird auch in Europa geführt - wiederum

72 U.S. National Intelligence Report 2017.

73 U.S. National Intelligence Council 2021.

74 Dies waren Forschungsprojekte der Universitäten Oxford und Columbia, Canfield Research LLC und der Cybersecurity-Firma New Knowledge; siehe Scott Shane/Sheera Frenkel: Russian Election Effort Focused on Influencing African-American Vote., New York Times, 18.12.2018, A1. Zum Informationskrieg gegen den Westen mithilfe sozialer Netzwerke siehe Bodine-Baron/Helmus/Radin/Treyger 2018, Spahn 2021 sowie Aro 2019 und 2021. sowohl verdeckt als auch im öffentlichen Raum. Zur ersteren Kategorie gehören Aktivitäten der russischen Geheimdienste, um die Unabhängigkeitsbewegung in Katalonien zu unterstützen, die politischen Verhältnisse in Moldova zu destabilisieren und einen Staatsstreich in Montenegro $\mathrm{zu}$ inszenieren. ${ }^{75}$ Indizien und Beweise gibt es auch für das Bemühen des Kremls, eine Abspaltung Schottlands vom Vereinigten Königreich und den Austritt Großbritanniens aus der EU (Brexit) zu befördern. ${ }^{76}$ Und Deutschland ist laut einer Untersuchung des Auswärtigen Dienstes der EU zum Hauptziel russischer Desinformationskampagnen geworden. ${ }^{77}$

Allerdings werfen die politisch und propagandistisch genutzten Angriffe von Hackern und Desinformationsund Destabilisierungsaktivitäten ein methodologisches Problem auf. Deren tatsächlicher Einfluss auf Entwicklungen im Westen ist kaum messbar. Zudem kann auch eine ganze Armee von aus Moskau gesteuerten Hackern, Trolls und Influenzern nicht urplötzlich breite politische gesellschaftliche Bewegungen hervorzaubern. Möglich ist allerdings, glühende Konfliktherde zu einem Großbrand zu entfachen - ein Phänomen, das beispielsweise auf die Wahl Trumps bis hin zum Angriff einer Meute auf das Kapitol am 6. Januar 2021 seinen (vorläufigen?) Höhepunkt gefunden hat. Oft haben auch nur geringe Prozentzahlen von Schwankungen schicksalhafte Konsequenzen. Ein wichtiges Beispiel dafür ist wiederum die US-Präsidentschaftswahl 2016. Berechnungen der Washington Post zufolge wäre die Wahl anders entschieden worden, wenn nur weniger als 80000 Stimmen in drei Einzelstaaten (Mi-

75 Michael Schwirtz: Secret Spy Unit In Russia Aims To Jolt Europe, New York Times, 9.10.2010, A1. Laut dem auf westlichen Geheimdienstinformationen basierenden Bericht spielte bei diesen Operationen die GRU-Einheit 29155 die zentrale Rolle. Diese Einheit soll auch für die Vergiftung der Skripals und den Mord an einem Waffenschmuggler in Bulgarien verantwortlich sein.

76 Zur Abspaltung Schottlands: Scottish Independence: „Russia attempted to influence 2014 referendum' reveals report, Scotsman. com, 21.7.2020, https://www.scotsman.com/news/politics/scottishindependence-russia-attempted-influence-2014-referendum-revealsreport-2919234. Ein Bericht, der auf dem Intelligence and Security Committee of Parliament Report 2020 beruht. Der Bericht fand zwar keine „Beweise“, greift aber die britische Regierung dafür an, dass diese sich nicht darum gekümmert habe, behaupteten Einmischungsversuchen überhaupt nachzugehen. $\mathrm{Zu}$ den Indizien für die Einmischung siehe u. a. Arron Banks „Met Russian officials multiple times before Brexit vote“, The Guardian 9.6.2018.

77 Vilifying Germany; Wooing Germany, Euvsdisinfo.eu, 9.3.21, https://euvsdisinfo.eu/villifying-germany-wooing-germany/. In ihrer Datenbank listet die Enthüllungsplattform der EU mehr als 700 Beispiele gezielter Desinformation in Deutschland seit Ende 2015 auf; siehe auch Spahn 2021. 
chigan, Pennsylvania und Wisconsin), also 0,06 Prozent der 137 Millionen abgegebenen Stimmen, nicht Trump, sondern Clinton zugefallen wären. ${ }^{78}$ Ähnlich verhält es sich mit dem Referendum in Großbritannien über den EU-Austritt im Juni 2016. Der Unterschied zwischen den für "Leave" und "Remain" abgegebenen Stimmen betrug lediglich 1,9 Prozent.

\section{Fazit}

Begriffe sollten nicht inhaltslos sein, keine Worthülsen oder Leerformeln. Sie sollten als Orientierungshilfe für Entscheidungsfindung dienen, aber auch verwandt werden, um einen breiteren öffentlichen Diskurs zu führen. Dies betrifft auch die Begriffe von strategischer Partnerschaft und strategischer Gegnerschaft. Der von der Bundesregierung und der EU gegen Ende der Jelzin-Ära gewählte Begriff der „strategischen Partnerschaft“" als Beschreibung der Beziehungen zu Russland war schon zu diesem Zeitpunkt realitätsfremd. Allerdings fügte er sich konzeptionell in die Vorstellung ein, dass Russland schon unumkehrbar den Weg zu einer Demokratie westlichen Zuschnitts, mit freier Marktwirtschaft, rechtstaatlichen Verhältnissen und einer aktiven Zivilgesellschaft im Inneren (transition theory) und in der Außenpolitik zur Zusammenarbeit mit dem Westen beschritten habe. Es war jedoch bereits in der Übergangsphase von Jelzin zu Putins erster Amtszeit als Präsident offensichtlich, dass die „Strategien“ Deutschlands und mutatis mutandis der EU - soweit man überhaupt davon sprechen konnte - und die Strategie Russlands weit auseinandergingen. Der Kreml spielte in der wirklichkeitsfremden Begriffswelt der „strategischen Partnerschaft“ zwar mit, verfolgte aber seine eigenen, Deutschland und der EU entgegenstehende Interessen und beschritt einen eigenen Entwicklungsweg. ${ }^{79}$

Dieser Weg führte von zunehmender Entfremdung zwischen dem offiziellen Russland und dem Westen zum Ende der Jelzin-Ära über ein Intervall, zumindest rhetorisch, mithilfe des Westens zu bewerkstelligender sozioökonomischer „Modernisierung“ während der Präsidentschaft Medwedews und schließlich seit dem Beginn der

78 Philip Bump: Donald Trump will be president thanks to 80,000 people in three states, Washington Post, 1.12.2016.

79 Das konnte man der Strategie Russlands gegenüber der EU in mittelfristiger Perspektive (2000-2010) entnehmen, einem Dokument, das Moskaus Antwort auf die Gemeinsame Strategie der EU gegenüber Russland darstellt. Putin übergab es der EU-,,Troika“ in seiner Eigenschaft als Regierungschef im Oktober 1999 in Helsinki: Mittelfristige Strategie Russlands 1999. dritten Amtszeit Putins als Präsident zu national-patriotischer Mobilisierung und Konfrontation mit dem Westen. Diese Entwicklung ist nicht als zusammenhanglose Anhäufung von Konflikten, geschweige denn Missverständnissen und Fehlwahrnehmungen $\mathrm{zu}$ verstehen, sondern als eine prinzipielle, nachhaltige und innenpolitisch in der Machtstruktur des Systems Putin verankerte und von der Moskauer Machtelite bewusst herbeigeführte Orientierung. ${ }^{80}$ Entsprechend hat sich das Verhältnis zwischen Russland und dem Westen von der Illusion einer bereits existierenden „strategischen Partnerschaft“ zur Realität einer strategischen Gegnerschaft entwickelt.

Diese stellt die zentrale Legitimationsbasis des Putin Systems dar. Sie wird nicht „teilweise“, sondern umfassend, in allen Bereichen der Politik geführt. Zentrales Instrument ist dabei, wie schon in der Sowjetära, militärische Macht, ihre Anwendung oder ihre Androhung. Ebenso wie im Kalten Krieg bilden Öl, Gas und andere Rohstoffe eine weitere Machtbasis und sind außenpolitische Instrumente. Die ideologische Komponente hat sich seit der Sowjetära zwar verändert, aber als Teil (wie es heute heißt) „hybrider Kriegsführung bemüht sich der Kreml wie ehedem, Konflikte und (wie es damals hieß) „Widersprüche“ zwischen den „imperialistischen Machtzentren“ USA und Europa, sowie zwischen und in den europäischen Ländern zu nutzen und, wo immer möglich, zu schüren.

In westlicher Perzeption wird diese Realität zum großen Teil weiterhin geleugnet, weniger in den USA als in Europa, und im östlichen Europa weniger als in Deutschland und dem südlichen Europa. In der Bundesregierung gibt es zwar ein geschärftes Bewusstsein dafür, dass das „feindselige“ Verhalten des Kremls einem „Muster“ folgt und integraler Bestandteil einer „Strategie hybrider Kriegsführung“ ist. Es besteht aber weiterhin eine große Kluft zwischen derartigen - oft nur sporadisch und verhalten, nicht nachdrücklich und systematisch vorgetragenen - Charakterisierungen und der gegenüber Moskau

80 Auf das prinzipielle und langfristige Wesen der Entfremdung als eine der Voraussetzungen für die Gegnerschaft hat - in Umkehrung der Prozesse, die dazu geführt haben - Vladislav Surkov, ein enger Vertrauter und Berater Putins, wie folgt hingewiesen: „Russlands epische Reise in den Westen ist vorbei. [Es] hat das Ende seiner wiederholten fruchtlosen Versuche über vier Jahrhunderte hinweg, ein Teil der westlichen Zivilisation zu werden und sich mit der, guten Familie‘ europäischer Nationen zu verheiraten, erreicht und tritt in eine neue Ära geopolitischer Einsamkeit ein, die 100 - vielleicht 200?, 300? - Jahre dauern kann. “ Allerdings bedeute dies nicht, dass Russland eine isolationistische Politik verfolgen würde. Siehe Vladislav Surkov: Odinočestvo polukrovki, Rossija v global'noj politike [Russland in der Weltpolitik], Globalaffairs Online, 11.4.2018, http://www. globalaffairs.ru/number/-19490. 
verfolgten Politik. Es ist in der Tat „höchste Zeit, dass wir eine strategische Debatte führen" "${ }^{81}$ mit politischen Folgerungen, die der Realität strategischer Gegnerschaft mit Russland Rechnung trägt.

Viele Politiker und Politikerinnen in Deutschland begnügen sich mit der Verurteilung der „Völkerrechtswidrigkeit“" von Handlungen Russlands, insbesondere auf der Krim. Und immer wieder wird dann die Forderung laut, dass man Russland besser verstehen und gegebenenfalls auf Moskau „zugehen“ müsse. Derartige Formeln verdrängen die Tatsache, dass die umfassende Gegnerschaft Russlands in Moskau gewollt ist und daher eine strategische Realität darstellt, die eine strategische Antwort der westlichen Staatengemeinschaft verlangt. Die Entwicklung der Beziehungen Russlands zum Westen ist integraler Bestandteil einer „Zeitenwende“ in den internationalen Beziehungen, die in all ihren damit verbundenen Konsequenzen $\mathrm{zu}$ akzeptieren die politische Elite Deutschlands überfordert. ${ }^{82}$

\section{Literatur}

Adomeit, Hannes (2011): Russia and Its Neighbourhood: Conflict and Competition with the EU, College of Europe. Natolin: College of Europe (Natolin Campus, Research Papers, No. 4/2011)

Adomeit, Hannes (2012a): Integrationskonkurrenz EU-Russland, Osteuropa, 62 (6-8), 383-406

Adomeit, Hannes (2012b): Russlands Politik in Osteuropa: Konkurrenz und Konflikte mit der Europäischen Union. Wien: Internationales Institut für Liberale Politik

Adomeit, Hannes (2014): Die „Eurasische Union“ - Reintegration des postsowjetischen Raums oder Teil einer „Gemeinschaft von Lissabon bis Wladiwostok"? in: Eckart D. Stratenschulte (Hrsg.): Heilsame Vielfalt? Formen differenzierter Integration in Europa. Baden-Baden: Nomos, $225-274$

Adomeit, Hannes (2016): Russlands Grenzziehungen - Abgrenzung, Ausgrenzung und Grenzkorrekturen, in: Joachim Klose/Rüdiger Voigt (Hrsg.), Grenzen in Zeiten der Entgrenzung, Dresden: Thelem-Verlag, 63-81

Adomeit, Hannes (2017a): Innenpolitische Determinanten der Putinschen Außenpolitik, Sirius: Zeitschrift für Strategische Analysen, 1 (1), 33-52

Adomeit, Hannes (2017b): Altes Denken, statt Neues Russland: Innenpolitische Bestimmungsfaktoren der russischen Außenpolitik, Portal Politikwissenschaft, 26.September, https://www.pw-portal.de/putins-russland/40508-altesdenken-statt-neues-russland

Adomeit, Hannes (2017c): Auf dem Tiefpunkt: USA-Russland Beziehungen unter Trump und Putin, Osteuropa, 68 (5), 115-133

81 Roth 2021.

82 Vgl. zur Zeitenwende Krause 2017.
Adomeit, Hannes (2019): Müssen wir Russland besser verstehen lernen? Eine kritische Auseinandersetzung mit den Argumenten für eine neue Russlandpolitik, Sirius: Zeitschrift für strategische Analysen, 3 (3), 224-241

Allison, Roy (2013): Russia and Syria: explaining alignment with a regime in crisis, International Affairs, 89 (4), 795-823

Aro, Jessikka (2019): Putin Trollit [Putin's Trolls: On the Frontlines of Russia's Information War Against the World], Helsinki: Elina Ahlback.

Aro, Jessikka (2021): Emilia Seikkanen Worked in a Trendy Video Start-Up in Berlin - Tells All about the Kremlin's Global Information Operation, Finnish Public Service Media Company, Yle.fi; https://yle.fi/uutiset/3-11820154

Bilban, Christoph/Gringinger, Hanna (2019): Mythos „GerasimovDoktrin “: Ansichten des russischen Militärs oder Grundlage hybrider Kriegsführung? Wien: Schriftenreihe der [österreichischen] Landesverteidigungsakademie

Bodine-Baron, Elizabeth/Helmus, Todd C./Radin, Andrew/Treyger, Elina (2018): Countering Russian Social Media Influence, Santa Monica, Cal.: RAND Corporation

Connolly, Richard/Boulège, Mathieu (2018): Russia's New State Armament Programme: Implications for the Russian Armed Forces and Military Capabilities to 2007. London: Royal Institute for International Affairs

Gady, Franz-Stefan (2019): Are Russia and China the Entente Cordiale of the 21st Century? New York: East-West Institute

Gerasimov, Valerij V. (2013): Cennost' nauki v predvidenii, VoennoPromyšlennyj Kur'er, Vpk-news.ru, 27. Februar, http:// vpk-news.ru/sites/default/files/pdf/VPK_08_476.pdf.

Gobel, Paul (2020): Russia, Iran Expand Military Cooperation Against US and Europe in Gulf, Eurasia Daily Monitor, 17 (137)

Gorenburg, Dmitry (2020): An Emerging Strategic Partnership: Trends in Russia-China Military Cooperation. Garmisch-Partenkirchen: George C. Marshall European Center for Security Studies

Gressel, Gustav (2018): Russian manoeuvres with Chinese characteristics. Berlin: European Council on Foreign Relations

Ilssaev, Leonid (2021): Russia's „Return“ to the Middle East and the Arab Uprisings. Istanbul: Al Sharq Forum

Kaczmarski, Marcin (2018): The Decreasing Asymmetry in RussiaChinese Relations: The Consequences of the Western Policy Shift towards China. Helsinki: Finnish Institute of International Affairs

Klein, Margarete (2016): Russlands neue Nationalgarde: Eine Rückversicherung für Putin gegen Massenproteste und illoyale Eliten. Berlin: Stiftung Wissenschaft und Politik (SWP-Aktuell 55)

Kolesnikov, Andrej (2020): Erinnerung als Waffe; Die Geschichtspolitik des Putin-Regimes, Osteuropa, 70 (6), 3-28

Krause, Joachim (2017): Die neue Zeitenwende in den internationalen Beziehungen - Konsequenzen für deutsche und europäische Politik, Sirius - Zeitschrift für strategische Analysen, 1 (1), 3-24

Makocki, Michal/Popescu, Nicu (2016): China and Russia: An Eastern Partnership in the Making? Paris: European Institute for Security Studies (Chaillot Paper No. 140)

Mössinger, Kira (2020): Russian disinformation and strategic narratives against the West. A case study of Russia's instrumentalization of the migrant crisis during Germany's 
Bundestag election. Leiden, NL.: University of Leiden, MA Thesis, Russian and Eurasian Studies

Paul, Michael (2019): Allianz auf hoher See? Chinas und Russlands gemeinsame Marinemanöver. Berlin: Stiftung Wissenschaft und Politik (SWP-Aktuell 24)

Quiring, Manfred (2017): Putins russische Welt: Wie der Kreml Europa spaltet. Berlin: Ch. Links Verlag

Spahn, Susanne (2021): Russische Medien in Deutschland: Wie der russische Informationskrieg und Desinformation Einfluss in Deutschland ausüben. Berlin: Friedrich-Naumann Stiftung für die Freiheit

Stewart, Susan (2020): Geschichte als Instrument der Innen- und Außenpolitik Russlands. Berlin: Stiftung Wissenschaft und Politik (SWP-Studie 22)

Stronski, Paul/Ng, Nicole (2018): Cooperation and Competition: Russia and China in Central Asia, the Russian Far East, and the Arctic. Washington, D.C.: Carnegie Endowment for International Peace

Wacker, Gudrun (2002): Chinesisch-russische Beziehungen unter Putin. Berlin: Stiftung Wissenschaft und Politik (SWP-Studie S 19)

Weiss, Andrew/Ng, Nicole (2019): Collision Avoidance: The Lessons of U.S. and Russian Operations in Syria. Washington, DC, Carnegie Endowment of International Peace

\section{Offizielle Veröffentlichungen und Dokumente}

Bundesministerium der Verteidigung (2021): Positionspapier: Gedanken zur Bundeswehr der Zukunft. Berlin: BMVG, https:// www.bmvg.de/resource/blob/5028534/44dcd6d650e6c1 f19ab2b82fe1f9510f/210209_BMin\%20\%26\%20GenInsp_ Positionspapier-Bundeswehr\%20der\%20Zukunft.pdf

Estonian Foreign Intelligence Service (2021): International Security and Estonia 2021. Tallinn: Estonian Foreign Intelligence Service, https://www.valisluureamet.ee/pdf/raport/2021-ENG. pdf

Europäische Union (1999): Gemeinsame Strategie der Europäischen Union gegenüber Russland. Amtsblatt der Europäischen Gemeinschaften, 1999/414/GASP, 24.6.1999

Intelligence and Security Committee of Parliament (2020): Russia Report [on interference in British politics] Cryptome.org, 21. Juli, https://cryptome.org/2020/07/ISC_Russia_Report.pdf Militärdoktrin der Russischen Föderation (2014): Voennaja doktrina Rossijskoj Federatsii, https://rg.ru/2014/12/30/doktrina-dok. html

Mittelfristige Strategie Russlands gegenüber der EU (1999): Strategija razvitija otnošenij Rossijskoj Federacii s Evropejskim Sojuzom na srednesročnuju perspektivu (2000-2010), Elektronisches Archiv „Techekspert“, Cntd.ru, http://docs.cntd.ru/ document/901773061

U.S. National Intelligence Council (2021): Foreign Threats to the 2020 U.S. elections. Declassified Document. Washington, D.C.: Office of the Director of National Intelligence, 10.3.2021

U.S. Department of Defense (2018): National Defense Strategy Summary. Washington, D.C.: Department of Defense

U.S. National Intelligence Report (2017): Background to "Assessing Russian Activities and Intentions in Recent US Elections": The Analytic Process and Cyber Incident Attribution. Washington, DC: Office of the Director of National Intelligence, Dni.gov, 6.1.2017, https://www.dni.gov/files/documents/ICA_2017_01. pdf 